\title{
Novel Modified Elman Neural Network Control for PMSG System Based on Wind Turbine Emulator
}

\author{
Chih-Hong Lin \\ Department of Electrical Engineering, National United University, Miao Li 360, Taiwan \\ Correspondence should be addressed to Chih-Hong Lin; jhlin@nuu.edu.tw
}

Received 24 December 2012; Accepted 22 March 2013

Academic Editor: Shengyong Chen

Copyright ( 2013 Chih-Hong Lin. This is an open access article distributed under the Creative Commons Attribution License, which permits unrestricted use, distribution, and reproduction in any medium, provided the original work is properly cited.

\begin{abstract}
The novel modified Elman neural network (NN) controlled permanent magnet synchronous generator (PMSG) system, which is directly driven by a permanent magnet synchronous motor (PMSM) based on wind turbine emulator, is proposed to control output of rectifier (AC/DC power converter) and inverter (DC/AC power converter) in this study. First, a closed loop PMSM drive control based on wind turbine emulator is designed to generate power for the PMSG system according to different wind speeds. Then, the rotor speed of the PMSG, the voltage, and current of the power converter are detected simultaneously to yield better power output of the converter. Because the PMSG system is the nonlinear and time-varying system, two sets online trained modified Elman NN controllers are developed for the tracking controllers of DC bus power and AC power to improve output performance of rectifier and inverter. Finally, experimental results are verified to show the effectiveness of the proposed control scheme.
\end{abstract}

\section{Introduction}

Since the petroleum is gradually exhausting and environmental protection is progressively rising, the usage of the clean energy sources such as wind, photovoltaic, and fuel cells has become very important and quite popular in electric power industries. Clean energy sources such as wind, photovoltaic, and fuel cells can be interfaced to a multilevel converter system for a high power application [1-3].

Wind turbine which acted as sources of energy has progressively increased in the whole earth. The various control methods and convert technologies of wind energy conversion systems are fast developed in energy conversion application. The PM synchronous generator system has been used for wind power generating system due to many advantages such as simpler structure, better reliability, lower maintenance, and higher efficiency [4-8]. Therefore, the PM synchronous generator generation system stands for a significant trend in progress of wind power applications [4-8]. The output power behavior of wind turbine is nonlinear. The provided power of vertical-axis turbines is very sensitive to the load variation due to different structure effect [4-8]. Thus, the control of operating point is indispensable for maximum output power. The controllable rectifier is used to convert varied AC voltage generated by PM synchronous generator into DC bus voltage. Then, the controllable inverter is used to convert DC bus voltage into $\mathrm{AC}$ at a fixed frequency in order to provide for the stand alone or grid applications of electrical utilizations. The major purposes of utilizing wind turbines are to extract maximum power of turbine and deliver appropriate energy to stand alone power or grid power. According to these purposes, the better structure of the power conversion in wind turbines is the AC to DC to AC power converter [912]. Reference [11] proposes the intelligent control of a windturbine emulator and an induction-generator (IG) system with an AC/DC power converter using a radial basis function network (RBFN). An on-line trained RBFN is developed for the tracking controller of DC-link power to improve the control performance. Reference [12] proposes a radial basis function network (RBFN) controlled three-phase IG system using $\mathrm{AC} / \mathrm{DC}$ and $\mathrm{DC} / \mathrm{AC}$ power converters. Two online trained RBFNs using backpropagation learning algorithm with improved particle swarm optimization (IPSO) are used as the regulating controllers for both the DC-link voltage and the $\mathrm{AC}$ line voltage of the $\mathrm{DC} / \mathrm{AC}$ power inverter. The IPSO is adopted in this study to adapt the learning rates in the backpropagation process of the RBFNs to improve the learning capability. 
The Elman neural network (NN) is a partial recurrent network model that was first proposed by Elman [13]. Typical Elman NN has one hidden layer with delayed feedback. The Elman NN is capable of providing the standard state-space representation for dynamic systems. The Elman NN can be considered to be a special type of recurrent neural network with feedback connections from the hidden layer to the context layer. The context layer is an additional layer that is used as an extra memory to memorize previous activations of the hidden neurons and to feed all the hidden neurons after the one-step time delay. Therefore, compared with the general recurrent neural networks, Elman NN has a special explicit memory to store the temporal information. Due to the context neurons, it has certain dynamical advantages over static neural network [14-16] and it also has been widely applied in dynamical systems identification and control [17-20]. Generally, Elman NN can be considered to be a special kind of feed-forward neural network with additional memory neurons [13]. Furthermore, the Elman NN can approximate high-order systems with high precision, and its converge speed is fast. Reference [20] proposes a fieldprogrammable gate array (FPGA)-based Elman NN control system to control the mover position of a linear ultrasonic motor (LUSM). A piecewise continuous function is adopted to replace the sigmoid function in the hidden layer of the ENN to facilitate implementation of the FPGA chip hardware in order to reduce cost and raise high performance.

The recurrent neural network has received increasing attention due to its structural advantage in nonlinear system modeling and dynamic system control [21-25]. The most important characteristic of the recurrent neural network is its self-connection to memorize feedback information of the historical influence in the same neuron. Moreover, in the general recurrent neural networks, the specific selfconnection feedback of the hidden neuron or output neuron is responsible for memorizing the specific previous activation of the hidden neuron or output neuron and feeding itself only. Therefore, the outputs of the other neurons have no ability to affect the specific neuron. However, in the complicated nonlinear dynamic system such as PMSG system directdriven by PMSM, the friction torque, various wind, and external nonlinear interference are always a factor. Hence, if each neuron in the recurrent neural networks is considered as a state in the nonlinear dynamic systems, the self-connection feedback type is unable to approximate the dynamic systems efficiently. On the other hand, the feedbacks in Elman NN not only are self-connecting but they also store in the context neurons and feed all the hidden neurons. Thus, the structure of Elman NN is more powerful than the general recurrent neural networks for dealing with time varying, and nonlinear dynamic systems can be approximated efficiently with the additional context layer. In order to improve the ability of identifying high-order systems, some modified Elman NN [26-28] have been proposed recently, which proved to have more advantages than the basic Elman NNs, including a better performance, higher accuracy, dynamic robustness, and a fast transient performance. The modified Elman neural network adopted in this paper has not only the feedback connection from the context layer in the hidden layer but also the delay feedback connection from the output layer in the input layer to raise control and transient performance.

Since the PMSGs have robust construction, lower initial and lower maintenance cost, PMSG are suitable for stand alone or grid power sources in small wind energy application. Therefore a PMSM direct-drive PMSG system using the two sets of the same modified Elman NN controllers is introduced as the adjusting controllers for both the DC bus voltage of the rectifier and the $\mathrm{AC} 60 \mathrm{~Hz}$ line voltage of the inverter in this study. Two online trained modified Elman NNs are introduced as the adjusting controllers for both the DC bus voltage of the controllable rectifier and the AC $60 \mathrm{~Hz}$ line voltage of the controllable inverter. Moreover, the training algorithms of two sets of the same online trained modified Elman NNs based on backpropagation are derived to train the recurrent weights, connective weights, translations, and dilations. Additionally, for the comparison of the control performance, the proportional integral (PI) controller can be also executed in the PMSG system. However, the control gains of the PI controller are obtained by trial and error method which is very time-consuming in practical applications. Due to the PMSG system with many uncertainties, adjusted capacity and tracking capacity of output voltage controlled by using the PI controller is less improved. To raise the desired robustness and overcome the above problem, the modified Elman NN controller is proposed to control output DC bus voltage of the rectifier produced by PMSM direct-driven PMSG system and control output voltage of the inverter provided by DC bus power. In the proposed modified Elman NN controller, the recurrent weights, connective weights, translations, and dilations are trained online via learning algorithm. Meanwhile, to demonstrate better dynamic characteristics of the proposed controller, comparative studies with the PI controller and the conventional NN controller are demonstrated by experimental results. Therefore, the control performance of the proposed modified Elman NN control system is much improved and can be verified by some experimental results.

This paper is organized as follows. The configuration of PMSG system is reviewed in Section 2. The novel modified Elman NN control system design is presented in Section 3. Experimental results are illustrated in Section 4. Some conclusions are given in Section 5.

\section{Configuration of PMSG System}

The variable speed wind turbine of the PMSG system directdriven by PMSM is a complex electromechanical system, which includes the mechanical components and the PMSG. The description of these components is presented as follows.

2.1. Model of Wind Turbine. The characteristic curve of the wind power versus rotor speed for model of wind turbine at different wind speeds in steady state shown in Figure 1 is very important for PMSG system direct-driven by PMSM. The power specification of the adopted wind turbine is the $1.5 \mathrm{~kW}$ in this paper. Its diameter is $2 \mathrm{~m}$. It is the three-blade horizontal axis type. It is capable of obtaining the working point of the wind turbine that used the intersection point of the load characteristic curve and the turbine characteristic 


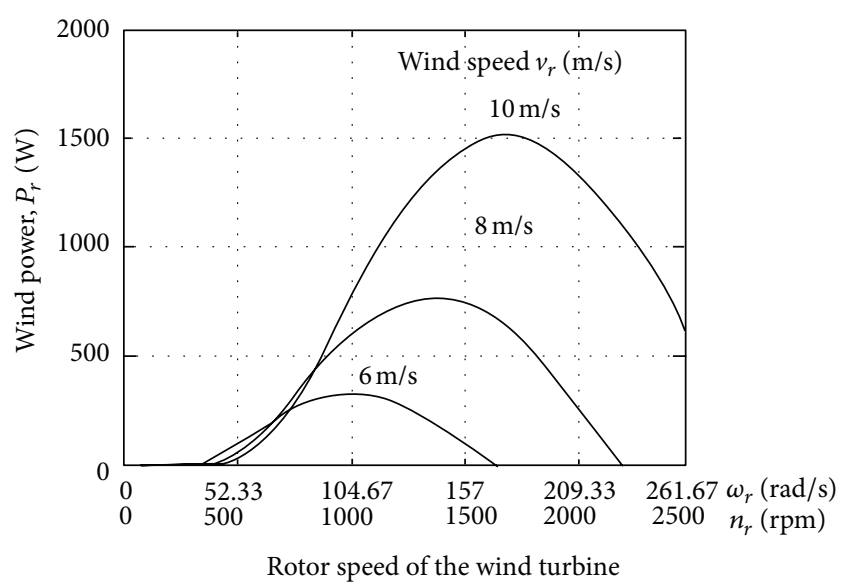

FIGURE 1: Characteristic curves of wind power versus rotor speed for wind turbine model at different wind speeds.

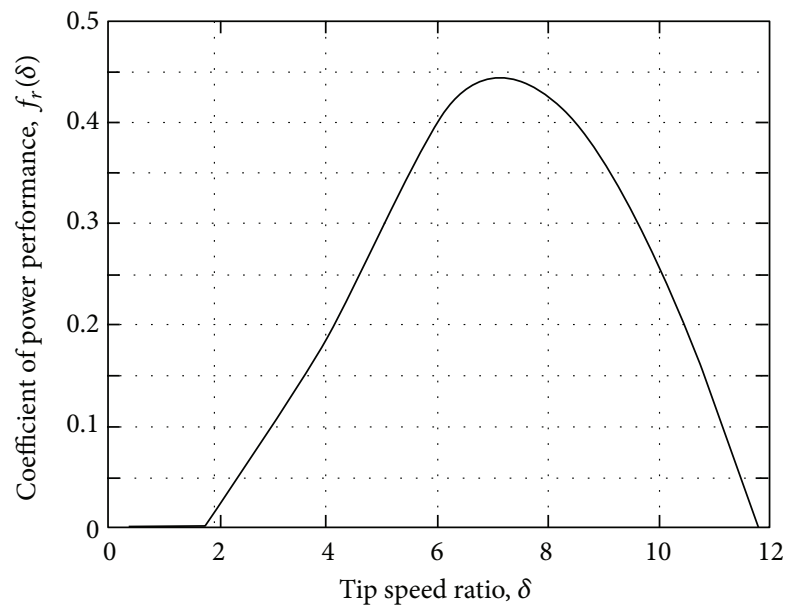

FIGURE 2: Characteristic curve of coefficient $f_{r}(\delta)$ of power performance versus tip ratio $\delta$ for wind turbine.

curve at a designated wind speed. It is a very important characteristic curve that the shaft power of the wind turbine relates to the wind speed $v_{1}$ and rotor speed $\omega_{r 1}$ to the maximum power tracking in Figure 1. For convenient usage and application, the characteristic curve shown in Figure 2 can also be expressed as the characteristic curve of the wind turbine model. It represents relationship curve between coefficient $f_{r}(\delta)$ of power performance and tip speed ratio $\delta$. According to aerodynamic principle $[1,4-7]$, the tip speed ratio $\delta$ of the wind turbine can be represented as

$$
\delta=\frac{d_{r} \omega_{r}}{v_{r}}
$$

where $\delta$ is the tip speed ratio of the wind turbine, $d_{r}$ is the rotor radius of the wind turbine in meter, $\omega_{r}$ is the rotor speed of the wind turbine in $\mathrm{rad} / \mathrm{s}$, and $v_{r}$ is the wind speed in $\mathrm{m} / \mathrm{s}$. The output mechanical power $P_{r}$ of the wind turbine can be expressed as [1, 4-7]

$$
P_{r}=\frac{\rho_{r} A_{r} f_{r}(\delta) v_{r}^{3}}{2}=\frac{\rho_{r} A_{r} f_{r}(\delta) d_{r}^{3} \omega_{r}^{3}}{\left(2 \delta^{3}\right)},
$$

where $\rho_{r}$ is the density of air in $\mathrm{kg} / \mathrm{m}^{3}$, and $A_{r}$ is the undraped area in $\mathrm{m}^{2}$. The generated torque of the wind turbine for different wind speeds can be obtained from the $f_{r}(\delta)-\delta$ curve in modeling usage. It is a very important case that the aerodynamic efficiency is maximum at the optimum tip speed ratio. The produced torque of the wind turbine can be indicated as follows $[1,4-7]$ :

$$
T_{r}=\frac{P_{r}}{\omega_{r}}=\frac{\rho_{r} A_{r} f_{r}(\delta) v_{r}^{3}}{\left(2 \omega_{r}\right)}=\frac{\rho_{r} A_{r} d_{r}^{3} f_{r}(\delta) \omega_{r}^{2}}{\left(2 \delta^{3}\right)},
$$

where $T_{r}$ is the produced torque of the wind turbine in $\mathrm{N}$ $\mathrm{m}$. A block diagram for the wind turbine model is shown in Figure 3. The block diagram shown in Figure 3 is to clearly describe the function between the input variable and output variable of the model.

Since the rotor of the wind turbine and the rotor of the PMSG are directly coupled through iron coupler, the mechanical angular speed of the rotor of the wind turbine is the same as the mechanical angular speed of the rotor of the PMSG for neglecting the stiffness of the wind turbine and the PMSG. From [29], the mechanical dynamic equation of torque, which the produced torque $T_{r}$ of the wind turbine subtract to the electromagnetic torque $T_{\mathrm{er}}$ of the PMSG, can be represented as

$$
\begin{gathered}
T_{r}-T_{\mathrm{er}}=J_{r} \frac{d \omega_{r}}{d t}+B_{r} \omega_{r}, \\
\omega_{\mathrm{er}}=\frac{P \omega_{r}}{2},
\end{gathered}
$$

where $J_{r}$ is the total moment of inertia in the direct-decoupled system of the wind turbine and the PMSG; $B_{r}$ is the total viscous friction coefficient in the direct-decoupled system of the wind turbine and the PMSG, $\omega_{\mathrm{er}}$ is the electrical angular speed of rotor, and $P$ is the number of poles of the PMSG.

2.2. Wind Turbine Emulator Based on PMSM. The wind turbine emulator, which is proposed in [4-10], is adopted in this study in order to emulate the wind turbine. Additionally, the adopted field-oriented controlled PMSM can be emulated the power speed characteristic curve of a wind turbine in this paper. In addition, a closed-loop robust speed controller, which can fight the intrinsic nonlinear and time-varying characteristic of the PMSM drive, is adopted to adjust the rotor speed with the relevant wind speed in order to emulate the wind variation.

2.3. Field-Oriented Controlled PMSG System. The voltage equations for the PMSG in the rotating reference frame can be indicated as follows [1, 7-10]:

$$
\begin{gathered}
v_{q 1}=-R_{s 1} i_{q 1}-L_{q 1} \dot{i}_{q 1}-\omega_{\mathrm{er}} L_{d 1} i_{d 1}+\omega_{\mathrm{er}} \lambda_{\mathrm{pm}} \\
v_{d 1}=-R_{s 1} i_{d 1}-L_{d 1} \dot{i}_{d 1}+\omega_{\mathrm{er}} L_{q 1} i_{q 1},
\end{gathered}
$$

where $v_{d 1}$ is the $d$-axis stator voltage, $v_{q 1}$ is the $q$-axis stator voltage, $i_{d 1}$ is the $d$-axis stator current, $i_{q 1}$ is the $q$-axis stator current, $L_{d 1}$ is the $d$-axis stator inductance, $L_{q 1}$ is the $q$-axis 


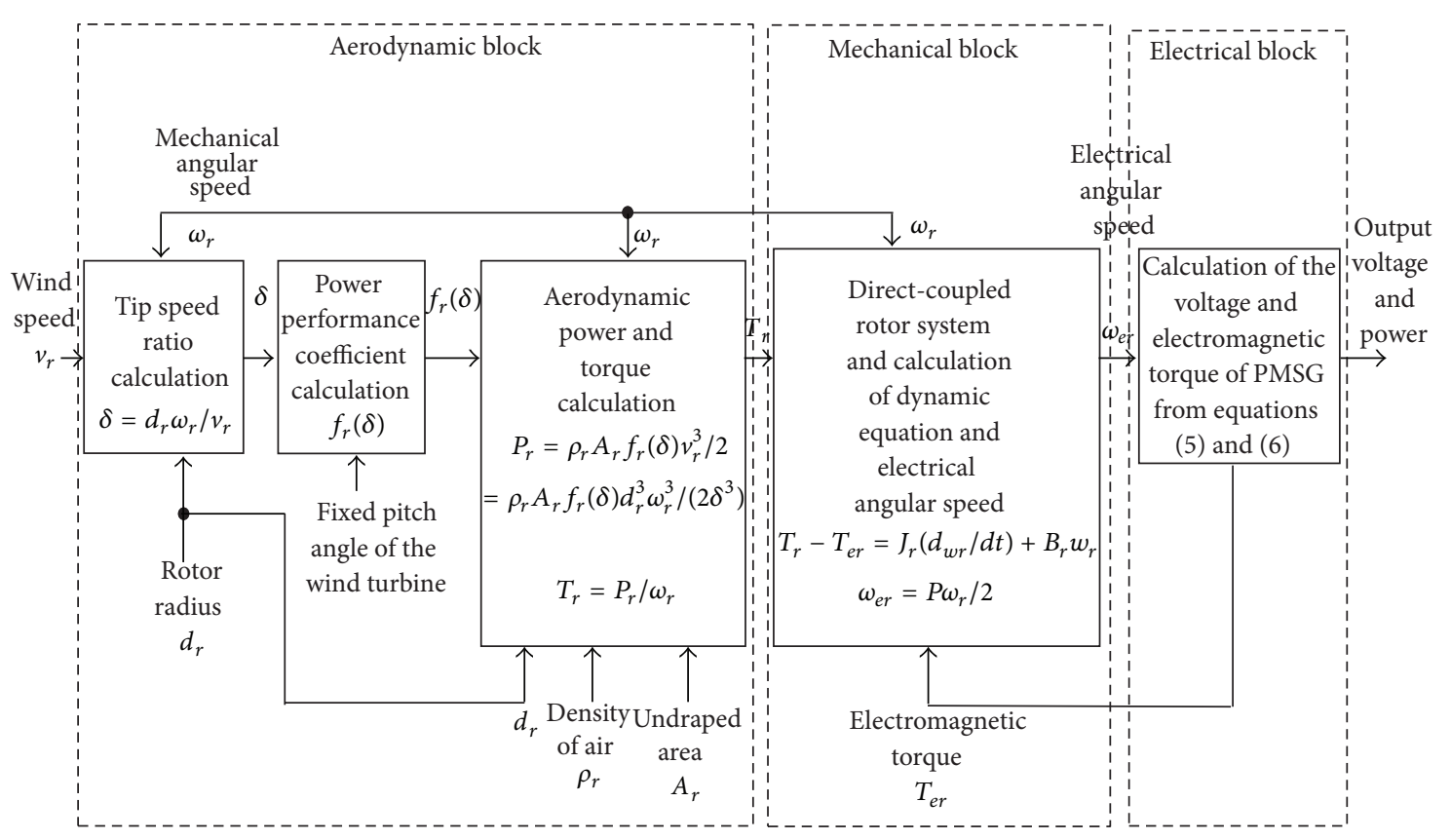

FIgURE 3: Block diagram for the wind turbine model.

stator inductance, and $R_{s 1}$ is the stator resistance. A fieldoriented control is adopted in [7-10]. By using the fieldoriented control, the $d$-axis stator current can be set zero, that is, $i_{d}=0$. Moreover, the electromagnetic torque of the PMSG can be expressed as

$$
T_{\mathrm{er}}=\frac{3}{2} \frac{P}{2}\left[\lambda_{\mathrm{pm}} i_{q 1}-\left(L_{d 1}-L_{q 1}\right) i_{d 1} i_{q 1}\right]=\frac{3}{4} \lambda_{\mathrm{pm}} i_{q 1}=K_{t} i_{q 1},
$$

where $\lambda_{\mathrm{pm}}$ is the permanent magnet flux linkage, and $K_{t}=$ $3 P \lambda_{\mathrm{pm}} / 4$ is the torque constant. For convenient analysis, the field-oriented controlled PMSG system is adopted. To emulate the operation of the wind turbine, the primary machine has adopted PM synchronous motor which directly mounted to PMSG. The control principle of the PMSG system is based on field orientation. Due to $L_{d 1}=L_{q 1}$ and $i_{d 1}=0$ in PMSG system, the second term of (6) is zero. Moreover, $\lambda_{\mathrm{pm}}$ is constant for a field orientation control of PMSG system. The electromagnetic torque $T_{\mathrm{er}}$ is a function of $i_{q 1}$. The electromagnetic torque $T_{\mathrm{er}}$ is linearly proportional to $q$ axis current $i_{q 1}$. When the $d$-axis rotor flux is constant, the maximum electromagnetic torque per ampere can be reached for the field-oriented control at the $T_{\text {er }}$ proportional to the $i_{q 1}$.

2.4. PMSG System. The control block diagram of the two sets of the same four-layer modified Elman NNs controlled PMSM direct-driven PMSG system is shown in Figure 4. The wind pattern can be acquired by appropriately programming the PMSM speed. The AC power of variable frequency and voltage generated by the PMSG system is rectified to DC power by controllable rectifier. The power converts of the PMSG system direct-driven by a PMSM consist of two field-oriented institutions, two current control loops, two sine pulse-width-modulation (SPWM) control circuits, two interlock and isolated circuits, and two IGBT power modules for rectifier and inverter. The DC bus voltage of the PMSG system directly driven by a PMSM via controllable rectifier can be controlled by using the first of modified Elman NN controller. Then, the inverter, which is controlled by using the second of modified Elman NN controller based on fieldoriented control, can convert the DC bus voltage into the AC $60 \mathrm{~Hz}$ line voltage to provide for the stand-alone load. The specification of PMSG is a three-phase four-pole $1.5 \mathrm{~kW}$ $220 \mathrm{~V} 10 \mathrm{~A} 2000 \mathrm{rpm}$ type for experimental test in this study. The electric parameters of the PMSG are $R_{s 1}=0.2 \Omega, L_{d 1}=$ $L_{q 1}=6 \mathrm{mH}$, and $L_{m}=6.2 \mathrm{mH}$. The specification of three-phase PMSM which acted as prime machine is a $1 \mathrm{~kW}$ $220 \mathrm{~V} 7 \mathrm{~A} 2000 \mathrm{rpm}$ type. In practical applications, the pure differentiator may amplify the high-frequency noise, so the stability of the closed-loop PMSG drive system will be greatly affected. Thus, a filter is implemented as an alternative for the pure differentiators shown in Figure 4. It is designed to behave as a pure differentiator for the main low-frequency dynamic signal and become a low-pass filter for the highfrequency signals.

The output voltages of rectifier and inverter controlled by two sets of the same modified Elman NN controllers were implemented by using two independent sets TMS320C32 DSP control system in Figure 4, where $\theta_{r}$ is the rotor position of the PMSG; $i_{d r}^{*}$ is the $d$ axis control current of the rectifier; $i_{q r}^{*}$ is the $q$ axis e control current of the rectifier; $i_{a r}^{*}, i_{b r}^{*}$ and $i_{c r}^{*}$ are the desired phase currents of the PMSG in phases $a r, b r$, and $c r$, respectively; $i_{a r}, i_{b r}$, and $i_{c r}$ are the actual measured phase currents of the PMSG in phases ar, br, and $c r$, respectively; $T_{a r}, T_{b r}$, and $T_{c r}$ are the SPWM control signals of the rectifier in phases $a r, b r$, and $c r$, respectively; $V_{d}$ is the actual measured magnitude of the DC bus voltage in output end of the rectifier; $V_{d}^{*}$ is the desired magnitude of the DC 


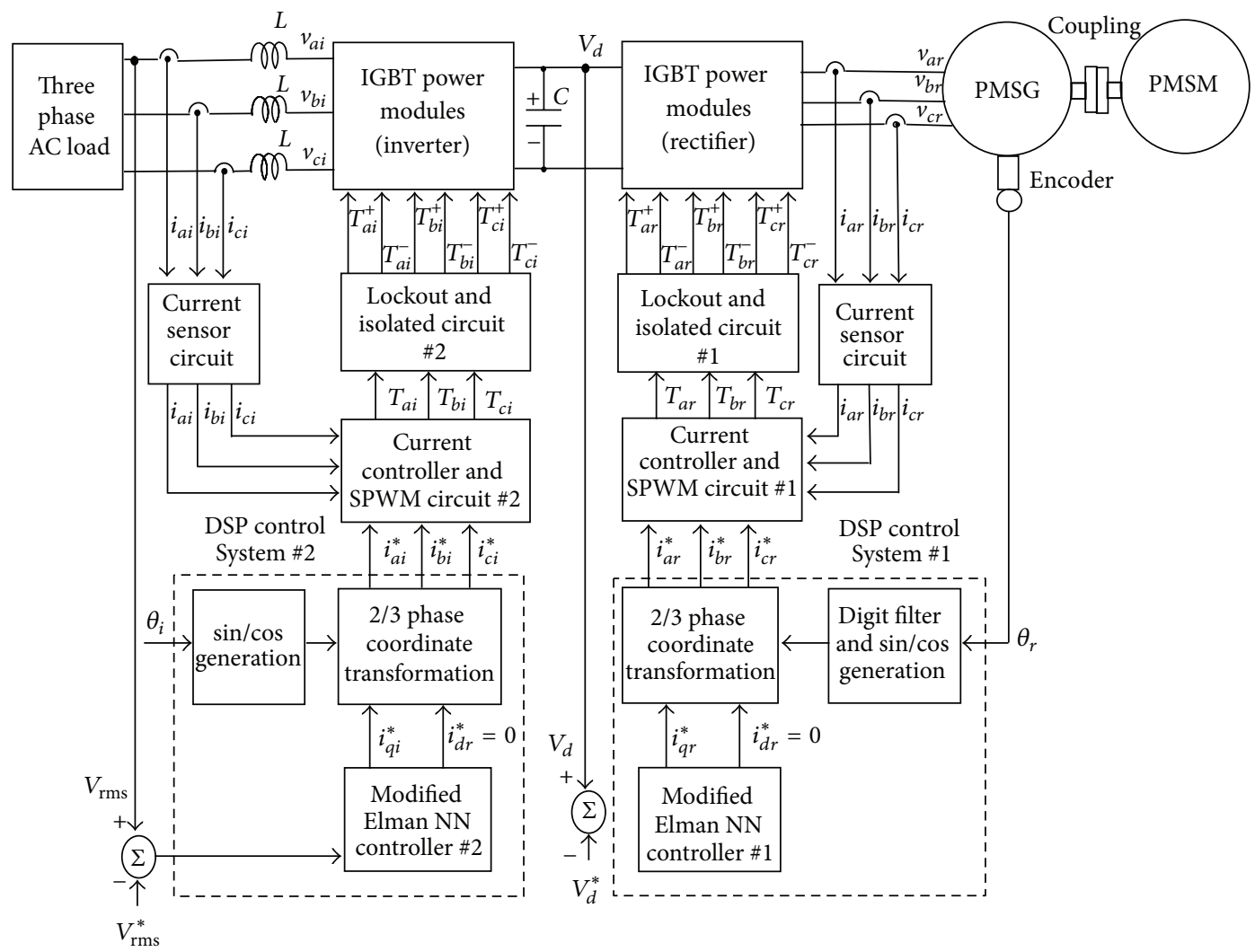

FIGURE 4: System configuration of the two sets of the same modified Elman NNs controlled PMSG system direct-driven by PMSM with rectifier and inverter.

bus voltage in output end of the rectifier; $i_{d i}^{*}$ is the $d$ axis control current of the inverter; $i_{q i}^{*}$ is the $q$ axis control current of the inverter; $\theta_{i}$ is the electric angular angle of the inverter which integrates the command electric angular frequency with respect to $t ; i_{a i}^{*}, i_{b i}^{*}$, and $i_{c i}^{*}$ are the desired phase currents of the inverter in phases $a i, b i$, and $c i$, respectively; $i_{a i}, i_{b i}$, and $i_{c i}$ are the actual measured phase currents of the inverter in phases $a i, b i$, and $c i$, respectively; $v_{a i}, v_{b i}$, and $v_{c i}$ are the actual measured phase voltages of the inverter in phases $a i$, $b i$, and $c i$, respectively; $T_{a i}, T_{b i}$, and $T_{c i}$ are the SPWM control signals of the inverter in phases $a i, b i$, and $c i$, respectively; $V_{\text {rms }}$ is the actual root-mean-square magnitude of the AC $60 \mathrm{~Hz}$ line voltage in output end of the inverter; $V_{\mathrm{rms}}^{*}$ is the desired root-mean-square magnitude of the AC $60 \mathrm{~Hz}$ line voltage in output end of the inverter.

\section{Novel Modified Elman NN Controller}

3.1. Description of Modified Elman NN. In the proposed two sets of the same four-layer modified Elman NNs with input layer using feedback signals from output layer are taken into account to result in better learning efficiency. The architecture of the two sets of the same four-layer modified Elman NNs, which consists of the first layer (the input layer), the second layer (the hidden layer), the third layer (the context layer), and the forth layer (the output layer), is shown in Figure 5. The exciting functions and signal propagations of nodes in

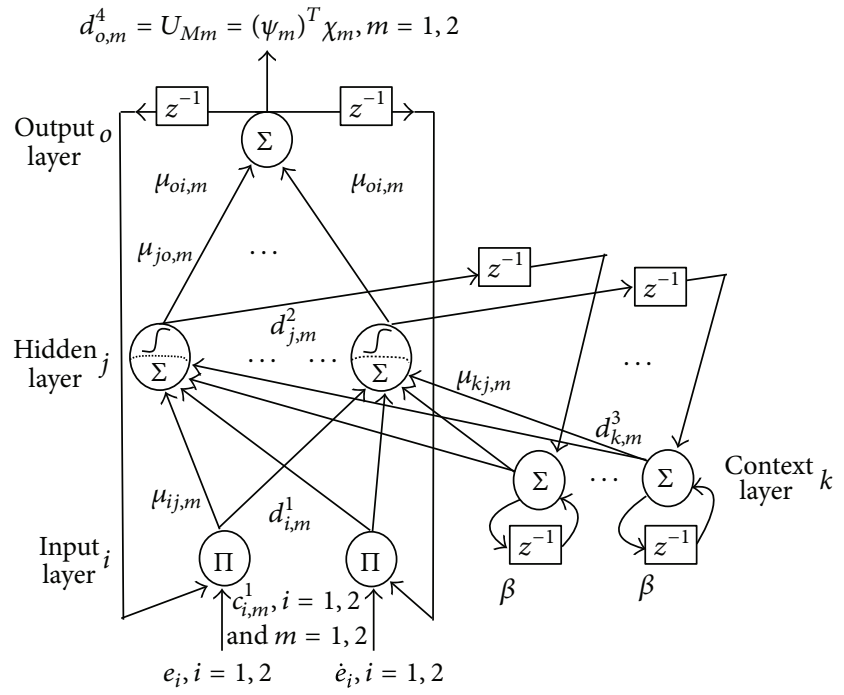

FIGURE 5: Structure of the two sets of the same four-layer novel modified Elman NNs.

each layer of the modified Elman NN can be described as follows.

3.1.1. First Layer: Input Layer. Each node $i$ in this layer is indicated by using, $\Pi$ which multiplies by each other between each other for input signals. Then outputs signals are 
the results of product. The input and the output for each node $i$ in this layer are expressed as

$$
\begin{aligned}
\operatorname{nod}_{i, m}^{1}(N) & =\prod_{o} c_{i, m}^{1}(N) \cdot \mu_{o i, m} \cdot d_{o, m}^{4}(N-1), \\
d_{i, m}^{1}(N) & =g_{i, m}^{1}\left(\operatorname{nod}_{i, m}^{1}(N)\right) \\
& =\operatorname{nod}_{i, m}^{1}(N), \quad i=1,2, \quad m=1,2,
\end{aligned}
$$

where $c_{i, m}^{1}$ is the input of the $i$ th nod in the $m$ th modified Elman NN, and $d_{i, m}^{1}$ is the output of the $i$ th nod in the $m$ th modified Elman NN. The different inputs of the two sets of modified Elman NNs are $c_{1,1}^{1}=e_{1}=V_{d}^{*}-V_{d}, c_{2,1}^{1}=\dot{e}_{1}$ for the rectifier end of the PMSG system in the first modified Elman $\mathrm{NN}$, and $c_{1,2}^{1}=e_{2}=V_{\mathrm{rms}}^{*}-V_{\mathrm{rms}}, c_{2,2}^{1}=\dot{e}_{2}$ for the inverter end of the PMSG system in the second modified Elman NN, respectively. The $N$ indicates the number of iterations. The connecting weights $\mu_{o i, m}$ are the recurrent weights between the output layer and the input layer in the $m$ th modified Elman NN. $d_{o, m}^{4}$ is the output value of the output layer in the $m$ th modified Elman NN.

3.1.2. Second Layer: Hidden Layer. The single node $j$ th in this layer is labeled with $\Sigma$. It computes outputs of the input layer and the context layer as the summation of all input signals. The net input and the net output for node $j$ th in this layer are expressed as

$$
\begin{aligned}
\operatorname{nod}_{j, m}^{2}(N) & =\sum_{k} \mu_{k j, m} d_{k, m}^{3}(N)+\sum_{i} \mu_{i j, m} d_{i, m}^{1}(N), \\
d_{j, m}^{2}(N) & =g_{j, m}^{2}\left(\operatorname{nod}_{j, m}^{2}(N)\right) \\
& =\frac{1}{1+e^{-\operatorname{nod}_{j, m}^{2}(N)}}, \quad j=1, \ldots, n, m=1,2,
\end{aligned}
$$

where $\mu_{k j, m}$ are the connective weights between the context layer and the hidden layer in the $m$ th modified Elman NN, $\mu_{i j, m}$ are the connective weights between the input layer and the hidden layer in the $m$ th modified Elman $\mathrm{NN}$, and $n$ is the number of neurons in the hidden layer; $g_{j, m}^{2}$ is the activation function in the $m$ th modified Elman NN, which is also a sigmoid function; $d_{i, m}^{1}(N)=c_{i, m}^{2}(N)$ represents the $i$ th output node of input layer in the $m$ th modified Elman $N N$, and $d_{k, m}^{3}(N)=c_{k, m}^{3}(N)$ represents the $k$ th input to the node of context layer in the $m$ th modified Elman NN.

3.1.3. Third Layer 3: Context Layer. In the context layer, the node input and the node output are represented as

$$
\begin{gathered}
\operatorname{nod}_{k, m}^{3}(N)=d_{j, m}^{2}(N-1)+\beta d_{k, m}^{3}(N-1), \\
d_{k}^{3}(N)=g_{k}^{3}\left(\operatorname{nod}_{k}^{3}(N)\right)=\operatorname{nod}_{k}^{3}(N), \quad k=1, \ldots, l,
\end{gathered}
$$

where $d_{j, m}^{2}(N)=c_{j, m}^{4}(N)$ represents the $j$ th output node of hidden layer in the $m$ th modified Elman NN; $d_{k, m}^{3}(N)$ represents the $k$ th output to the node of context layer in the $m$ th modified Elman NN; $l$ is the number of neurons in the context layer; $0 \leq \beta<1$ is the self-connecting feedback gain of context layer.
3.1.4. Fourth Layer: Output Layer. The single node oth in this layer is labeled with $\Sigma$. It computes the overall output as the summation of all input signals. The net input and the net output for node oth in this layer are expressed as

$$
\begin{gathered}
\operatorname{nod}_{o, m}^{4}(N)=\sum_{j} \mu_{j o, m} c_{j, m}^{4}(N), \\
d_{o, m}^{4}(N)=g_{o, m}^{4}\left(\operatorname{nod}_{o, m}^{4}(N)\right)=\operatorname{nod}_{o, m}^{4}, \quad o=1, m=1,2,
\end{gathered}
$$

where $\mu_{j o, m}$ are the connective weights between the hidden layer and the output layer in the $m$ th modified Elman NN; $g_{o, m}^{4}$ is the activation function in the $m$ th modified Elman $\mathrm{NN}$, which is set to be unit; $d_{j, m}^{2}(N)=c_{j, m}^{4}(N)$ represents the $j$ th input to the node of output layer in the $m$ th modified Elman NN. The outputs in the $m$ th recurrent wavelet NN can be represented as $d_{o, m}^{4}$

$$
d_{o, m}^{4}=\left(\psi_{m}\right)^{T} \chi_{m}, \quad m=1,2 .
$$

The output values of the two sets of the same fourlayer modified Elman NNs can be rewritten as $U_{M 1}=$ $\left(\psi_{1}\right)^{T} \boldsymbol{\chi}_{1}=i_{q r}^{*}$ for rectifier and $U_{M 2}=\left(\psi_{2}\right)^{T} \chi_{2}=i_{q i}^{*}$ for inverter. Two vectors $\psi_{1}=\left[\begin{array}{lllll}\mu_{11,1}^{4} & \mu_{21,1}^{4} & \cdots & \mu_{l 1,1}^{4}\end{array}\right]^{T}$ and $\psi_{2}=\left[\begin{array}{llll}\mu_{11,2}^{4} & \mu_{21,2}^{4} & \cdots & \mu_{l 1,2}^{4}\end{array}\right]^{T}$ are to be adjusted parameters between the mother layer and the output layer of the two sets of the same four-layer modified Elman NNs. The $\chi_{m}=\left[\begin{array}{llll}c_{1, m}^{4} & c_{2, m}^{4} & \cdots & c_{l, m}^{4}\end{array}\right]^{T}, \quad m=1,2$ are the inputs vectors in the output layer of the two sets of the same fourlayer modified Elman NNs, in which $c_{j, m}^{4}$ are determined by the selected sigmoid function and $0 \leq c_{j, m}^{4} \leq 1$.

3.2. Online Learning Algorithm of Modified Elman NN. To explain the online learning algorithm of the modified Elman NN using supervised gradient decent method, firstly the energy function $V_{c, m}$ is defined as

$$
V_{c, m}=\frac{1}{2} e_{m}^{2}, \quad m=1,2
$$

where $e_{1}$ equals to $V_{d}^{*}-V_{d}$ in rectifier end of the PMSG system; $e_{2}$ equals to $V_{\mathrm{rms}}^{*}-V_{\mathrm{rms}}$ in the inverter end of the PMSG system. Then, the learning algorithm is described as follows.

3.2.1. Fourth Layer. The propagated error term in the $m$ th modified Elman NN is

$$
v_{m}^{4}=-\frac{\partial V_{c, m}}{\partial d_{o, m}^{4}} \frac{\partial d_{o, m}^{4}}{\partial \operatorname{nod}_{o, m}^{4}}, \quad m=1,2
$$

Then variation $\Delta \mu_{j o, m}$ of connective weights in the $m$ th modified Elman NN can be calculated as

$$
\begin{aligned}
\Delta \mu_{j o, m} & =-\gamma_{1, m} \frac{\partial V_{c, m}}{\partial d_{o, m}^{4}} \frac{\partial d_{o, m}^{4}}{\partial \operatorname{nod}_{o, m}^{4}} \frac{\partial \operatorname{nod}_{o, m}^{4}}{\partial \mu_{j o, m}} \\
& =\gamma_{1, m} \cdot v_{m}^{4} \cdot c_{j, m}^{4}, \quad m=1,2,
\end{aligned}
$$


where $\gamma_{1, m}$ is the learning rate between hidden layer and output layer in the $m$ th modified Elman NN. The connective weights $\mu_{j o, m}$ between hidden layer and output layer in the $m$ th modified Elman NN can be renewed according to the following equation:

$$
\mu_{j o, m}(N+1)=\mu_{j o, m}(N)+\Delta \mu_{j o, m}, \quad m=1,2 .
$$

3.2.2. Second Layer. The propagated error term in the $m$ th modified Elman NN is

$$
\begin{aligned}
v_{j, m}^{2} & \triangleq-\frac{\partial V_{c, m}}{\partial d_{o, m}^{4}} \frac{\partial d_{o, m}^{4}}{\partial \operatorname{nod}_{o, m}^{4}} \frac{\partial \operatorname{nod}_{o, m}^{4}}{\partial d_{j, m}^{2}} \frac{\partial d_{j, m}^{2}}{\partial \operatorname{nod}_{j, m}^{2}} \\
& =v_{m}^{4} P_{j, m}(N), \quad m=1,2,
\end{aligned}
$$

where $P_{j, m} \equiv \partial d_{j, m}^{2} / \partial \operatorname{nod}_{j, m}^{2}$ can be calculated from (8).

Then variation $\Delta \mu_{k j, m}$ of connective weights in the $m$ th modified Elman NN can be calculated as

$$
\begin{aligned}
\Delta \mu_{k j, m} & =-\frac{\partial V_{c, m}}{\partial d_{o, m}^{4}} \frac{\partial d_{o, m}^{4}}{\partial \operatorname{nod}_{o, m}^{4}} \frac{\partial \operatorname{nod}_{o, m}^{4}}{\partial d_{j, m}^{2}} \frac{\partial d_{j, m}^{2}}{\partial \operatorname{nod}_{j, m}^{2}} \frac{\partial \operatorname{nod}_{j, m}^{2}}{\partial \mu_{k j, m}} \\
& =v_{j, m}^{2} \cdot d_{k, m}^{3}, \quad m=1,2,
\end{aligned}
$$

where connective weights $\mu_{k j, m}$ between context layer and hidden layer in the $m$ th modified Elman $\mathrm{NN}$ can be renewed according to the following equation:

$$
\mu_{k j, m}(N+1)=\mu_{k j, m}(N)+\Delta \mu_{k j, m}, \quad m=1,2 .
$$

Then variation $\Delta \mu_{i j, m}$ of connective weights in the $m$ th modified Elman NN can be calculated as

$$
\begin{aligned}
\Delta \mu_{i j, m}= & -\frac{\partial V_{c, m}}{\partial \operatorname{nod}_{o, m}^{4}} \frac{\partial d_{o, m}^{4}}{\partial \operatorname{nod}_{o, m}^{4}} \frac{\partial \operatorname{nod}_{o, m}^{4}}{\partial d_{j, m}^{2}} \frac{\partial d_{j, m}^{2}}{\partial \operatorname{nod}_{j, m}^{2}} \\
& \times \frac{\partial \operatorname{nod}_{j, m}^{2}}{\partial \mu_{i j, m}}=v_{j, m}^{2} \cdot d_{i, m}^{1}, \quad m=1,2,
\end{aligned}
$$

where connective weights $\mu_{i j, m}$ between hidden layer and input layer in the $m$ th modified Elman NN can be updated as

$$
\mu_{i j, m}(N+1)=\mu_{i j, m}(N)+\Delta \mu_{i j, m}, \quad m=1,2 .
$$

Then variation $\Delta \mu_{o i, m}$ of connective weights in the $m$ th modified Elman NN by using the chain rule and the gradient descent method can be calculated as

$$
\begin{aligned}
\Delta \mu_{o i, m} & =-\frac{\partial V_{c, m}}{\partial \operatorname{nod}_{j, m}^{2}} \frac{\partial \operatorname{nod}_{j, m}^{2}}{\partial d_{i, m}^{1}} \frac{\partial d_{i, m}^{1}}{\partial \operatorname{nod}_{i, m}^{1}} \frac{\partial \operatorname{nod}_{i, m}^{1}}{\partial \mu_{o i, m}} \\
& =v_{j, m}^{2} \cdot Q_{j, m},
\end{aligned}
$$

where $Q_{j, m} \equiv \partial d_{j, m}^{2} / u_{o i, m}$ can be calculated from (7). The recurrent weights $\mu_{o i, m}$ between output layer and input layer in $m$ th modified Elman NN can be renewed according to the following equation:

$$
\mu_{o i, m}(N+1)=\mu_{o i, m}(N)+\Delta \mu_{o i, m}, \quad m=1,2 .
$$

Due to the uncertainty effect of the system dynamics, the accurate computation of the Jacobian $\partial V_{c, m} / \partial d_{o, m}^{4}, m=$ 1,2 in the PMSG system cannot be determined. To dispel the difficulty and endure the above matter, using the delta adaptation law [20] can raise the online learning capacity of the connective weights. Therefore, the delta adaptation law can be calculated as [20]

$$
v_{m}^{4}=e_{m}+\dot{e}_{m}, \quad m=1,2 .
$$

3.3. Convergence Analysis. Selection of the values for the learning-rate parameters has a significant effect on the network performance. In order to train the modified Elman NN effectively, the varied learning rate, which guarantee convergence of the output error based on the analyses of a discrete-type Lyapunov function, is derived in this section. The convergence analysis is to derive specific learning-rate parameter for specific types of network parameter to assure convergence of the output error [30].

Theorem 1. Let $\gamma_{m}$ be the learning-rate parameter of the mth modified Elman $N N$ and let $p_{\max , m}$ be defined as $p_{\max , m} \equiv$ $\max _{N}\left\|p_{m}(N)\right\|$, where $p_{m}(N)=\partial d_{o, m}^{4} / \partial \mu_{j o, m}$ in the mth modified Elman $N N$ and $\|\cdot\|$ is the Euclidean norm in $\mathfrak{R}^{n}$. The convergence is guaranteed if $\gamma_{1, m}$ is chosen as $\gamma_{1, m}=$ $\lambda_{1} /\left(p_{\max , m}^{2}\right)=\lambda_{1} / R_{u, m}$, in which $\lambda_{1}$ is a positive constant gain; $R_{u, m}$ is the number of nodes in hidden layer of the mth modified Elman NN.

Proof. Since

$$
p_{m}(N)=\frac{\partial d_{o, m}^{4}}{\partial \mu_{j o, m}}=c_{j, m}^{4} .
$$

Thus

$$
\left\|p_{m}(N)\right\|<\sqrt{R_{u, m}}
$$

Then, a discrete-type Lyapunov function is selected as

$$
V_{m}(N)=\frac{1}{2} e_{m}^{2}(N) .
$$

The change in the Lyapunov function is obtained by

$$
\begin{aligned}
\Delta V_{m}(N) & =V_{m}(N+1)-V_{m}(N) \\
& =\frac{1}{2}\left[e_{m}^{2}(N+1)-e_{m}^{2}(N)\right] .
\end{aligned}
$$

The error difference can be represented by [30]

$$
\begin{aligned}
e_{m}(N+1) & =e_{m}(N)+\Delta e_{m}(N) \\
& =e_{m}(N)+\left[\frac{\partial e_{m}(N)}{\partial \mu_{j o, m}}\right]^{T} \Delta \mu_{j o, m},
\end{aligned}
$$


where $\Delta \mu_{j o, m}$ represents a weight change between hidden layer and output layer in the $m$ th modified Elman NN. Using (11), (12), (13), and (28), then

$$
\begin{gathered}
\frac{\partial e_{m}(N)}{\partial \mu_{j o, m}}=\frac{\partial e_{m}(N)}{\partial d_{o, m}^{4}} \frac{\partial d_{o, m}^{4}}{\partial \mu_{j o, m}}=-\frac{v_{m}^{4}}{e_{m}(N)} p_{m}(N) \\
e_{m}(N+1)=e_{m}(N)-\left[\frac{v_{m}^{4}}{e_{m}(N)} P_{m}(N)\right]^{T} \gamma_{1, m} v_{m}^{4} P_{m}(N) .
\end{gathered}
$$

Then

$$
\begin{aligned}
& \left\|e_{m}(N+1)\right\| \\
& \quad=\left\|e_{m}(N)\left[1-\gamma_{1, m}\left(\frac{v_{m}^{4}}{e_{m}(N)}\right)^{2} P_{m}^{T}(N) P_{m}(N)\right]\right\| \\
& \leq\|e(N)\|\left\|1-\gamma_{1, m}\left(\frac{v_{m}^{4}}{e_{m}(N)}\right)^{2} P_{m}^{T}(N) P_{m}(N)\right\| .
\end{aligned}
$$

If $\gamma_{1, m}$ is chosen as $\gamma_{1, m}=\lambda_{1} /\left(P_{\max , m}^{2}\right)=\lambda_{1} / R_{u, m}$, the term $\left\|1-\gamma_{1, m}\left(v_{m}^{4} / e_{m}(N)\right)^{2} p_{m}^{T}(N) p_{m}(N)\right\|$ in (30) is less than 1 . Therefore, the Lyapunov stability of $V_{m}>0$ and $\Delta V_{m}<0$ is guaranteed. The output error between the reference model and the actual system will converge to zero as $t \rightarrow \infty$. This completes the proof of the theorem.

Remark 2. The values of the learning-rate parameter $\gamma_{1, m}$ is dependent on the selection of the value $\lambda_{1}$.

\section{Experimental Results}

The two sets of the same modified Elman NNs controlled PMSG system are realized in two sets TMS320C32 DSP control system. A photo of the experimental setup is shown in Figure 6. To implement current controlled PWM rectifier and inverter by field-oriented control, the two sets IGBT power modules are adopted BSM 100 GB-120DLC manufactured by Eupec Co. The switching frequency of both IGBT power modules is all $15 \mathrm{kHz}$. The two programs of the two sets TMS320C32 DSP control system used for executing the two sets of the same modified Elman NNs and online training of the two sets of the same modified Elman NNs need $2 \mathrm{~ms}$ sampling interval. The proposed methodology for the real-time control implementation consists of the two main programs and two interrupt service routines (ISRs) in the two sets of DSP control system as shown in Figure 7. In the main program \#1, parameters and input/output (I/O) initialization are processed first. Then, the interrupt interval for the ISR \#1 is set. After enabling the interrupt, the main program \#1 is used to monitor control data. The ISR \#1 with $2 \mathrm{~ms}$ sampling interval is used for reading the rotor position of the PM synchronous generator from encoder, reading mechanic torque from torque transducer, reading measured DC bus voltage and current from analog/digital (A/D) converters, calculating maximum DC bus power of the PM synchronous generator and DC bus power, and executing the modified Elman NN control system \#1. On the other
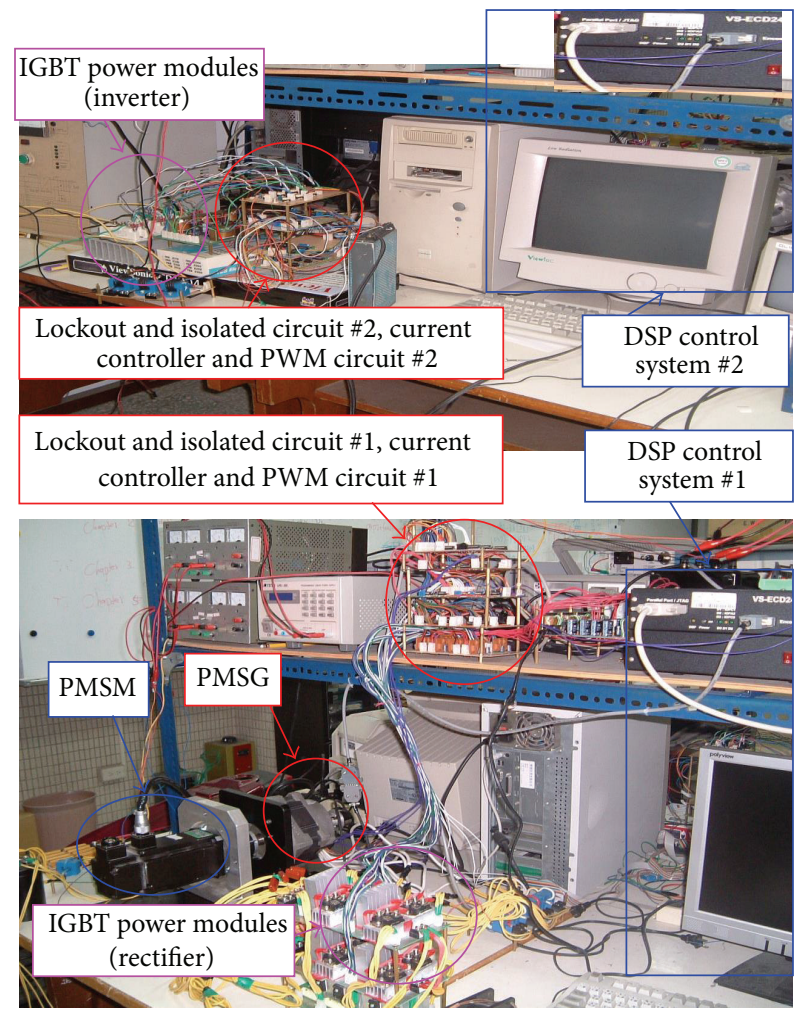

FIgURE 6: A photo of the experimental setup.

hand, in the main program \#2, parameters and input/output (I/O) initialization are processed first. Then, the interrupt interval for the ISR \#2 is set. After enabling the interrupt, the main program is used to monitor control data. The ISR \#2 with $2 \mathrm{~ms}$ sampling interval is used for reading the command angular frequency of sinusoidal wave to match demand of grid system, reading command root-mean-square AC line voltage and measured root-mean-square AC line voltage from analog/digital (A/D) converters, and executing the modified Elman NN control system \#2. Furthermore, to show the effectiveness of the control system, comparative studies with the PI controller and the conventional NN controller are demonstrated by experimental results. The conventional $\mathrm{NN}$ has 2, 5, and 1 nodes in the input layer, the hidden layer with sigmoid function, and the output layer, respectively. The modified Elman NN has 2, 5, 5, and 1 nodes in layer 1, layer 2, layer 3, and layer 4, respectively. The parameter adjustment process remains continually active for the duration of the experimentation. To verify the control performance of the proposed two sets of the same modified Elman NNs controlled PMSG system direct-driven by PMSM based on wind turbine emulator, three cases with the fieldoriented control current $i_{d i}^{*}=0 \mathrm{~A}$ and $i_{d r}^{*}=0 \mathrm{~A}$ are tested. To show the adjusting and tracking responses for the stand alone power application, three cases are selected. Firstly, case 1 is the $\Delta$ connection three-phase load $100 \Omega$, and the rotor speed $\omega_{r}\left(n_{r}\right)$, the step desired magnitude $V_{d}^{*}$ of DC bus voltage, and the step desired root-mean-square magnitude $V_{\mathrm{rms}}^{*}$ of AC $60 \mathrm{~Hz}$ line voltage are set as $78.5 \mathrm{rad} / \mathrm{s}(750 \mathrm{rpm}), 220 \mathrm{~V}$, and $110 \mathrm{~V}$, respectively. Secondly, case 2 is the $\Delta$ connection three-phase load $50 \Omega$ and the rotor speed $\omega_{r 1}$, the desired 

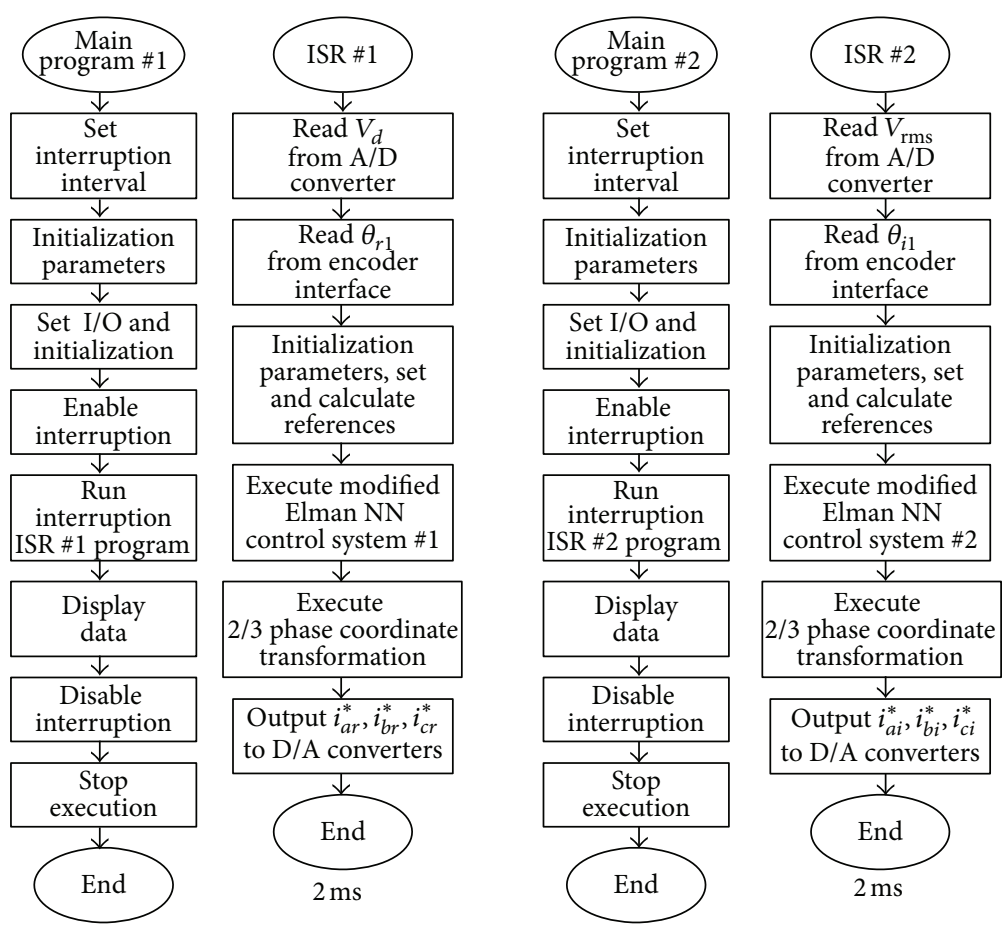

FIGURE 7: Flowcharts of two sets of the same modified Elman NN control systems controlled PMSG system.

magnitude $V_{d}^{*}$ of DC bus voltage, the desired root-meansquare magnitude $V_{\mathrm{rms}}^{*}$ of AC $60 \mathrm{~Hz}$ line voltage are set as $157 \mathrm{rad} / \mathrm{s}(1500 \mathrm{rpm}), 220 \mathrm{~V}$, and $110 \mathrm{~V}$, respectively. Thirdly, case 3 is the $\Delta$ connection three-phase load $18 \Omega$ and the rotor speed $\omega_{r}$, the desired magnitude $V_{d}^{*}$ of DC bus voltage, the desired root-mean-square magnitude $V_{\mathrm{rms}}^{*}$ of AC $60 \mathrm{~Hz}$ line voltage are set as $209.3 \mathrm{rad} / \mathrm{s}(2000 \mathrm{rpm}), 220 \mathrm{~V}$, and $110 \mathrm{~V}$, respectively. The $\Delta$ connection three-phase loads of $100 \Omega$, $50 \Omega$, and $18 \Omega$ dispatched powers as $121 \mathrm{~W}, 242 \mathrm{~W}$, and $672 \mathrm{~W}$, respectively. The $\Delta$ connection three-phase loads of $100 \Omega$ and $50 \Omega$, and $18 \Omega$ dispatched powers as $121 \mathrm{~W}, 242 \mathrm{~W}$, and $672 \mathrm{~W}$, respectively. Some experimental results of the PI controlled PMSG system direct-driven by PMSM are demonstrated for the comparison of the control performance. Since the PMSG system is a nonlinear time-varying system, the gains of the PI controllers for both the DC bus voltage adjustment and AC $60 \mathrm{~Hz}$ line voltage adjustment are obtained by trial and error to achieve steady state control performance. The control gains are $K_{p}=5.2, K_{i}=10.2$ for the DC bus voltage adjustment and $K_{p}=4.8, K_{i}=10.8$ for the $\mathrm{AC} 60 \mathrm{~Hz}$ line voltage adjustment in the two sets PI controllers. The experimental results of the PI controlled PMSG system direct-driven by PMSM for the $\Delta$ connection three-phase loads of $100 \Omega$ with $\omega_{r}=78.5 \mathrm{rad} / \mathrm{s}\left(n_{r}=750 \mathrm{rpm}\right)$ at case 1 , the $\Delta$ connection three-phase loads of $50 \Omega$ with $\omega_{r}=150 \mathrm{rad} / \mathrm{s}$ $\left(n_{r}=1500 \mathrm{rpm}\right)$ at case 2 , and the $\Delta$ connection three-phase loads of $18 \Omega$ with $\omega_{r}=209.3 \mathrm{rad} / \mathrm{s}\left(n_{r}=2000 \mathrm{rpm}\right)$ at case 3 are shown in Figures 8, 9 and 10, respectively; where rotor speed $\omega_{r}\left(n_{r}\right)$ is shown in Figures 8(a), 9(a) and 10(a); adjusting response of step desired magnitude $V_{d}^{*}$ of the DC bus voltage and actual measured magnitude $V_{d}$ of the DC bus voltage in output end of the rectifier is shown in Figures 8(b), 9(b), and 10(b); adjusting response of step desired rootmean-square magnitude $V_{\mathrm{rms}}^{*}$ of the AC $60 \mathrm{~Hz}$ line voltage and actual measured root-mean-square magnitude $V_{\text {rms }}$ of the AC $60 \mathrm{~Hz}$ line voltage in output end of the inverter is shown in Figures 8(c), 9(c), and 10(c); tracking response of the desired phase current $i_{a i}^{*}$ and actual measured phase current $i_{a i}$ in phase ai of the inverter is shown in Figures 8(d), 9 (d), and 10(d) respectively. From the experimental results, sluggish DC bus voltage and AC $60 \mathrm{~Hz}$ line voltage adjusting responses are obtained for the PI controlled PMSG system direct-driven by PMSM because of the weak robustness of the linear controller.

Some experimental results of the conventional NN controlled PMSG system direct-driven by PMSM for the $\Delta$ connection three-phase loads of $100 \Omega$ with $\omega_{r}=78.5 \mathrm{rad} / \mathrm{s}$ $\left(n_{r}=750 \mathrm{rpm}\right)$ at case 1 , the $\Delta$ connection three-phase loads of $50 \Omega$ with $\omega_{r}=150 \mathrm{rad} / \mathrm{s}\left(n_{r}=1500 \mathrm{rpm}\right)$ at case 2 , and the $\Delta$ connection three-phase loads of $18 \Omega$ with $\omega_{r}=209.3 \mathrm{rad} / \mathrm{s}\left(n_{r}=2000 \mathrm{rpm}\right)$ at case 3 are shown in Figures 11, 12, and 13, respectively, where rotor speed $\omega_{r}\left(n_{r}\right)$ is shown in Figures 11(a), 12(a), and 13(a); adjusting response of step desired magnitude $V_{d}^{*}$ of the DC bus voltage and actual measured magnitude $V_{d}$ of the DC bus voltage in output end of the rectifier is shown in Figures 11(b), 12(b), and 13(b); adjusting response of step desired rootmean-square magnitude $V_{\mathrm{rms}}^{*}$ of the $\mathrm{AC} 60 \mathrm{~Hz}$ line voltage and actual measured root-mean-square magnitude $V_{\text {rms }}$ of the $\mathrm{AC} 60 \mathrm{~Hz}$ line voltage in output end of the inverter is shown in Figures 11(c), 12(c), and 13(c); tracking response of the desired phase current $i_{a i}^{*}$ and actual measured phase current $i_{a i}$ in phase $a i$ of the inverter is shown in Figures 11(d), 12(d), and 13(d) respectively. From the experimental 


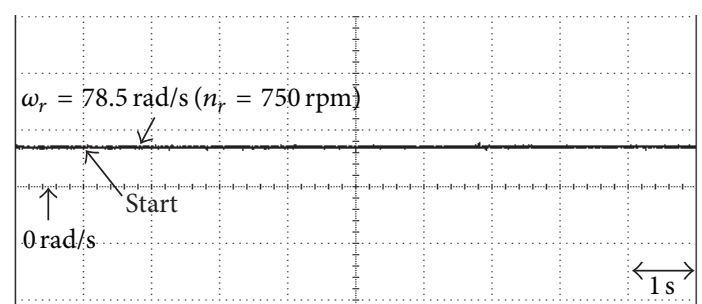

(a)

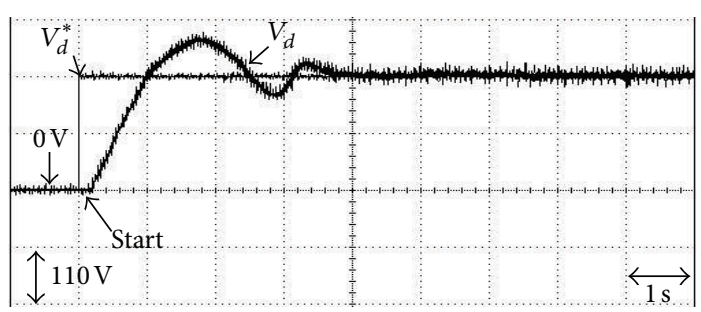

(b)

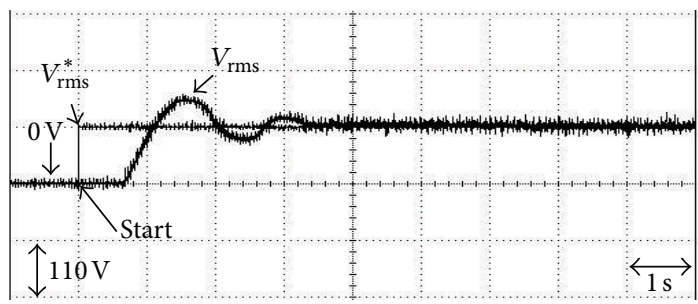

(c)

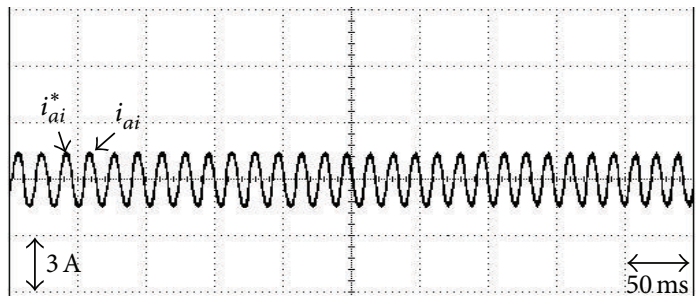

(d)

Figure 8: Experimental results of PMSM direct-driven PMSG system using the PI controller for the $\Delta$ connection three-phase loads of $100 \Omega$ with $\omega_{r}=78.5 \mathrm{rad} / \mathrm{s}\left(n_{r}=750 \mathrm{rpm}\right)$ at case 1: (a) rotor speed $\omega_{r}\left(n_{r}\right)$; (b) adjusting response of step desired magnitude $V_{d}^{*}$ of the DC bus voltage and actual measured magnitude $V_{d}$ of the DC bus voltage in output end of the rectifier; (c) adjusting response of step desired root-mean-square magnitude $V_{\mathrm{rms}}^{*}$ of the AC $60 \mathrm{~Hz}$ line voltage and actual measured root-mean-square magnitude $V_{\mathrm{rms}}$ of the AC $60 \mathrm{~Hz}$ line voltage in output end of the inverter $V_{\mathrm{rms}}$; (d) tracking response of the desired phase current $i_{a i}^{*}$ and actual measured phase current $i_{a i}$ in phase ai of the inverter.

results, few sluggish DC bus voltage and $\mathrm{AC} 60 \mathrm{~Hz}$ line voltage adjusting responses are obtained for the conventional NN controlled PM synchronous motor direct-drive PM synchronous generator system because the conventional NN is static input/output mapping schemes that can approximate a continuous function to an arbitrary degree of accuracy.

Some experimental results of the modified Elman NN controlled PMSG system direct-driven by PMSM for the $\Delta$ connection three-phase loads of $100 \Omega$ with $\omega_{r}=78.5 \mathrm{rad} / \mathrm{s}$

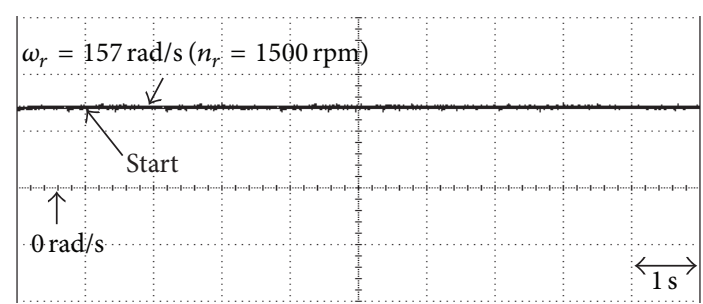

(a)

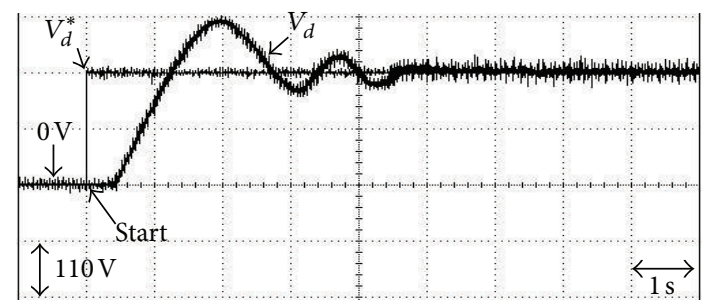

(b)

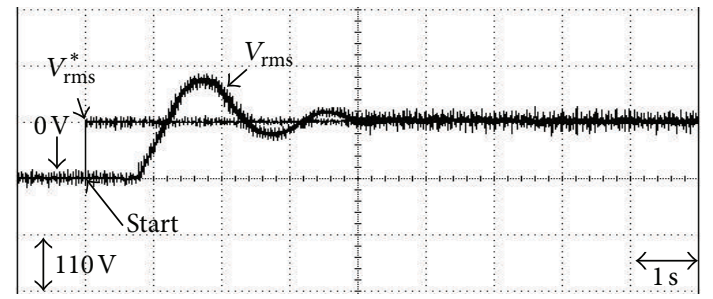

(c)

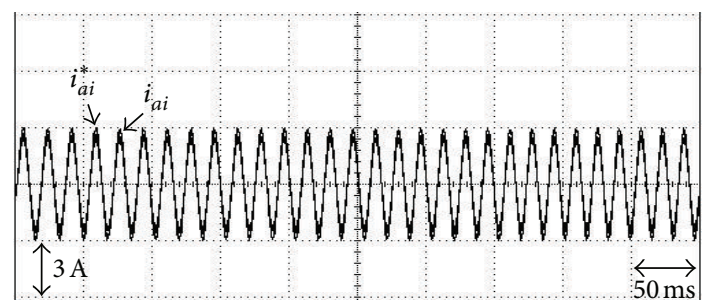

(d)

FIGURE 9: Experimental results of PMSM direct-driven PMSG system using the PI controller for the $\Delta$ connection three-phase loads of $50 \Omega$ with $\omega_{r}=150 \mathrm{rad} / \mathrm{s}\left(n_{r}=1500 \mathrm{rpm}\right)$ at case $2:$ (a) rotor speed $\omega_{r}\left(n_{r}\right)$; (b) adjusting response of step desired magnitude $V_{d}^{*}$ of the DC bus voltage and actual measured magnitude $V_{d}$ of the DC bus voltage in output end of the rectifier; (c) adjusting response of step desired root-mean-square magnitude $V_{\mathrm{rms}}^{*}$ of the AC $60 \mathrm{~Hz}$ line voltage and actual measured root-mean-square magnitude $V_{\text {rms }}$ of the AC $60 \mathrm{~Hz}$ line voltage in output end of the inverter $V_{\mathrm{rms}}$; (d) tracking response of the desired phase current $i_{a i}^{*}$ and actual measured phase current $i_{a i}$ in phase ai of the inverter.

( $\left.n_{r}=750 \mathrm{rpm}\right)$ at case 1 , the $\Delta$ connection three-phase loads of $50 \Omega$ with $\omega_{r}=150 \mathrm{rad} / \mathrm{s}\left(n_{r}=1500 \mathrm{rpm}\right)$ at case 2 , and the $\Delta$ connection three-phase loads of $18 \Omega$ with $\omega_{r}=209.3 \mathrm{rad} / \mathrm{s}$ $\left(n_{r}=2000 \mathrm{rpm}\right)$ at case 3 are shown in Figures 14, 15, and 16 , respectively; where rotor speed $\omega_{r}\left(n_{r}\right)$ is shown in Figures 14(a), 15(a), and 16(a); adjusting response of step desired magnitude $V_{d}^{*}$ of the DC bus voltage and actual measured magnitude $V_{d}$ of the DC bus voltage in output end of the rectifier is shown in Figures 14(b), 15(b), and 16(b); adjusting 


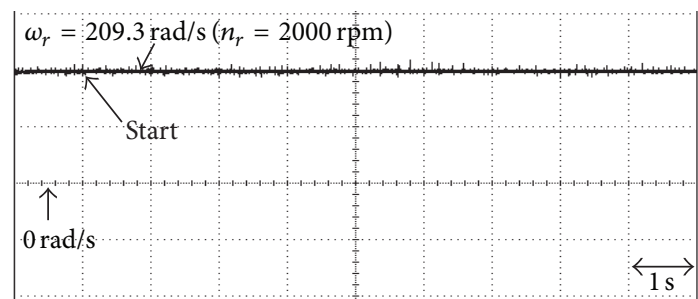

(a)

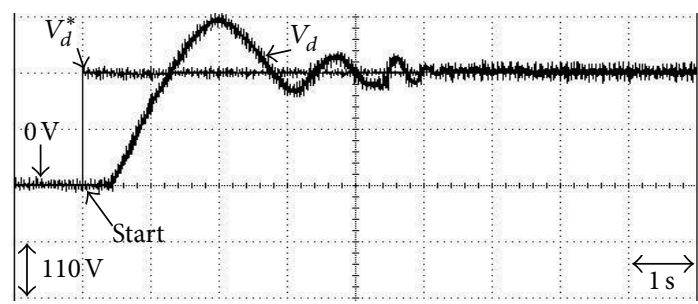

(b)

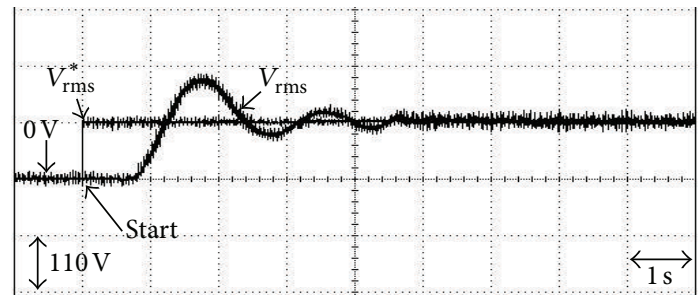

(c)

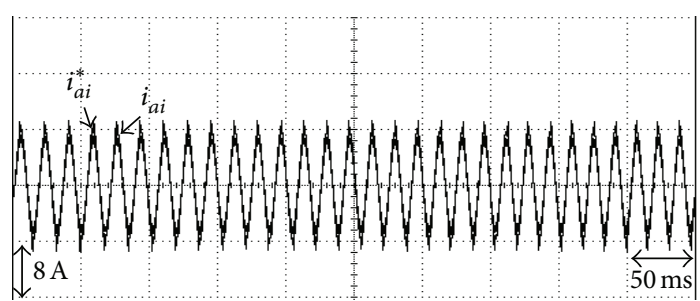

(d)

FIGURE 10: Experimental results of PMSM direct-driven PMSG system using the PI controller for the $\Delta$ connection three-phase loads of $18 \Omega$ with $\omega_{r}=209.3 \mathrm{rad} / \mathrm{s}\left(n_{r}=2000 \mathrm{rpm}\right)$ at case $3:$ (a) rotor speed $\omega_{r}\left(n_{r}\right)$; (b) adjusting response of step desired magnitude $V_{d}^{*}$ of the DC bus voltage and actual measured magnitude $V_{d}$ of the DC bus voltage in output end of the rectifier; (c) adjusting response of step desired root-mean-square magnitude $V_{\mathrm{rms}}^{*}$ of the AC $60 \mathrm{~Hz}$ line voltage and actual measured root-mean-square magnitude $V_{\text {rms }}$ of the AC $60 \mathrm{~Hz}$ line voltage in output end of the inverter $V_{\mathrm{rms}}$; (d) tracking response of the desired phase current $i_{a i}^{*}$ and actual measured phase current $i_{a i}$ in phase ai of the inverter.

response of step desired root-mean-square magnitude $V_{\mathrm{rms}}^{*}$ of the AC $60 \mathrm{~Hz}$ line voltage and actual measured root-meansquare magnitude $V_{\mathrm{rms}}$ of the AC $60 \mathrm{~Hz}$ line voltage in output end of the inverter is shown in Figures 14(c), 15(c), and 16(c); tracking response of the desired phase current $i_{a i}^{*}$ and actual measured phase current $i_{a i}$ in phase ai of the inverter is shown in Figures 14(d), 15(d), and 16(d), respectively. The overshoot and undershoot in DC bus voltage and AC $60 \mathrm{~Hz}$ line voltage at different rotor speeds by using modified Elman

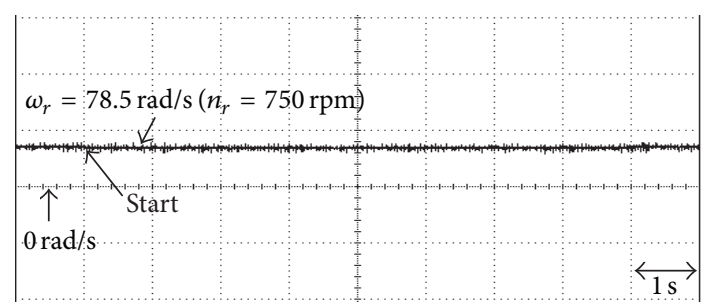

(a)

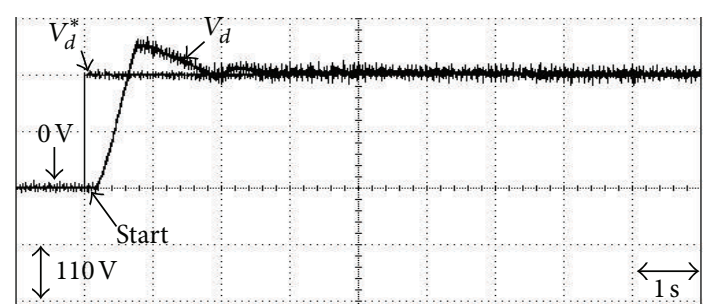

(b)

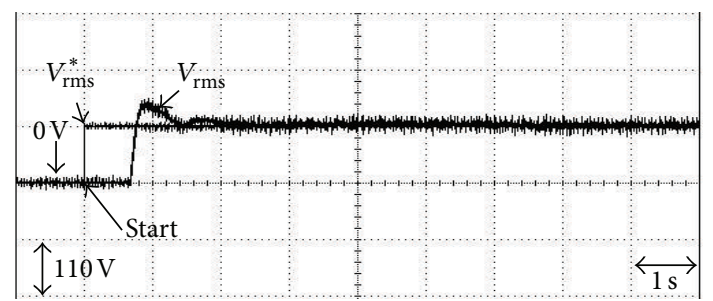

(c)

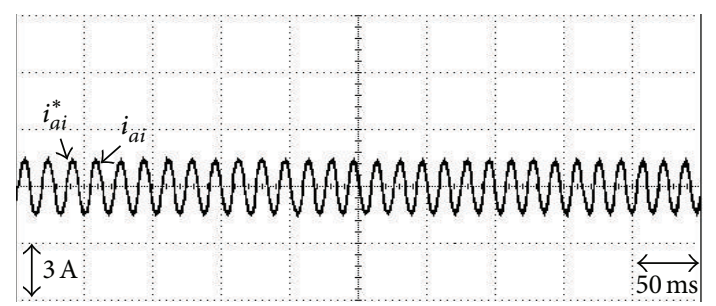

(d)

FIGURE 11: Experimental results of PMSM direct-driven PMSG system using the conventional NN controller for the $\Delta$ connection three-phase loads of $100 \Omega$ with $\omega_{r}=78.5 \mathrm{rad} / \mathrm{s}\left(n_{r}=750 \mathrm{rpm}\right)$ at case 1: (a) rotor speed $\omega_{r}\left(n_{r}\right)$; (b) adjusting response of step desired magnitude $V_{d}^{*}$ of the DC bus voltage and actual measured magnitude $V_{d}$ of the DC bus voltage in output end of the rectifier; (c) adjusting response of step desired root-mean-square magnitude $V_{\mathrm{rms}}^{*}$ of the AC $60 \mathrm{~Hz}$ line voltage and actual measured root-meansquare magnitude $V_{\mathrm{rms}}$ of the AC $60 \mathrm{~Hz}$ line voltage in output end of the inverter $V_{\text {rms }}$; (d) tracking response of the desired phase current $i_{a i}^{*}$ and actual measured phase current $i_{a i}$ in phase $a i$ of the inverter.

NN controllers as shown in Figures 14(b), 14(c), 15(b), 15(c), 16(b), and 16(c) are less magnitude and much improved by using the PI controller shown in Figures 8(b), 8(c), 9(b), 9(c), 10(b), and 10(c) and by using the conventional NN controller shown in Figures 11(b), 11(c), 12(b), 12(c), 13(b), and 13(c). Moreover, compared with the PI control and the conventional NN control, the proposed modified Elman NN control has much improved for the tracking ability and obviously reduced the oscillations in steady state. 


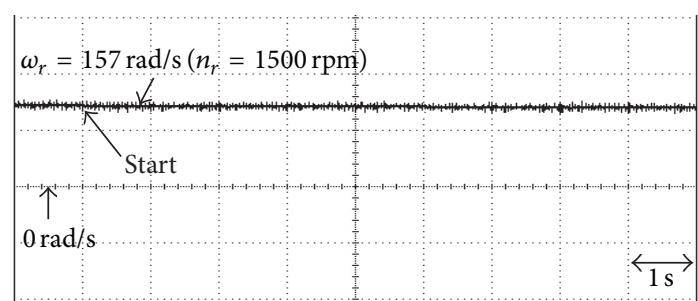

(a)

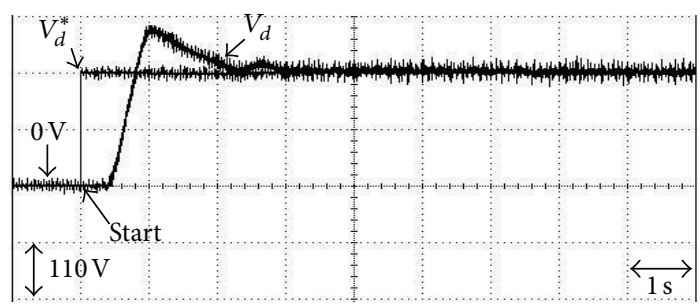

(b)

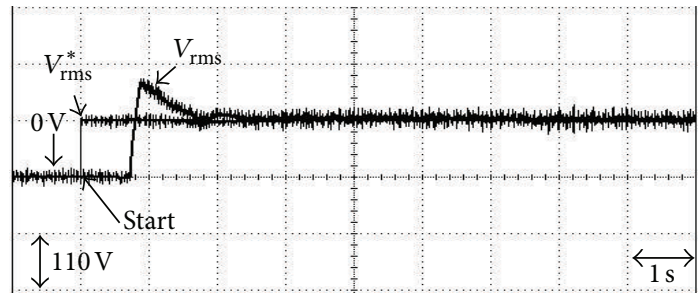

(c)

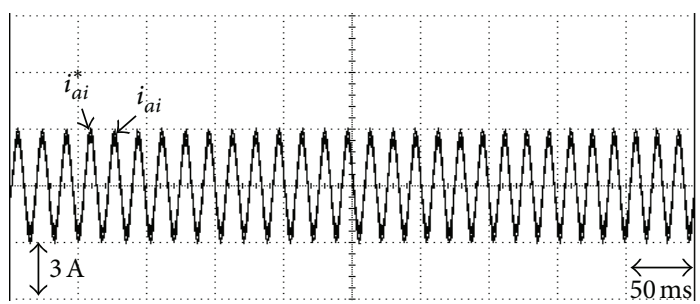

(d)

FIGURE 12: Experimental results of PMSM direct-driven PMSG system using the conventional NN controller for the $\Delta$ connection three-phase loads of $50 \Omega$ with $\omega_{r}=150 \mathrm{rad} / \mathrm{s}\left(n_{r}=1500 \mathrm{rpm}\right)$ at case 2: (a) rotor speed $\omega_{r}\left(n_{r}\right)$; (b) adjusting response of step desired magnitude $V_{d}^{*}$ of the DC bus voltage and actual measured magnitude $V_{d}$ of the DC bus voltage in output end of the rectifier; (c) adjusting response of step desired root-mean-square magnitude $V_{\mathrm{rms}}^{*}$ of the AC $60 \mathrm{~Hz}$ line voltage and actual measured root-meansquare magnitude $V_{\text {rms }}$ of the AC $60 \mathrm{~Hz}$ line voltage in output end of the inverter $V_{\mathrm{rms}}$; (d) tracking response of the desired phase current $i_{a i}^{*}$ and actual measured phase current $i_{a i}$ in phase ai of the inverter.

\section{Conclusions}

This study demonstrated the implementation of both the DC bus voltage and $\mathrm{AC} 60 \mathrm{~Hz}$ line voltage adjustment of the PMSG system direct-driven by PMSM based on wind turbine emulator by using the two sets of the same modified Elman NN controllers for stand alone power applications. Firstly, the field-oriented control was implemented for the control of the PMSG system direct-driven by PMSM based on wind turbine emulator. Then, the proposed two sets of the same

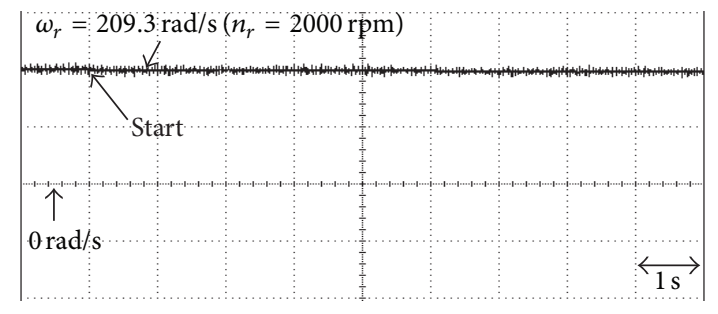

(a)

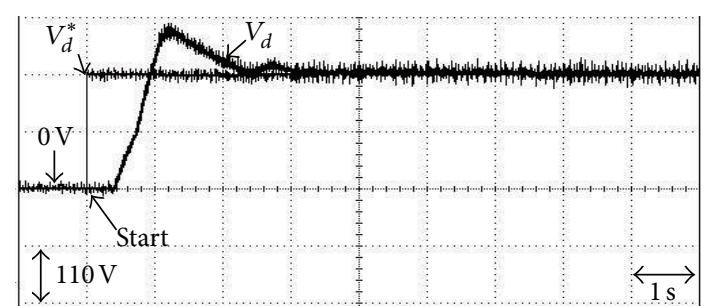

(b)

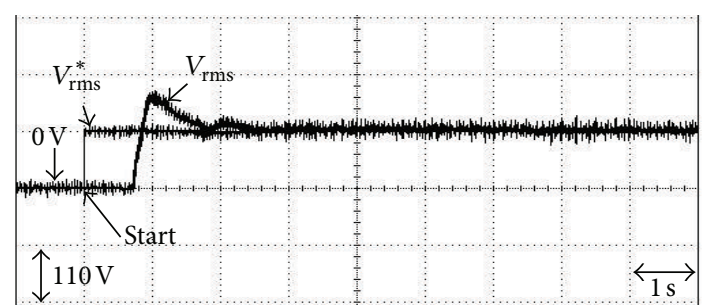

(c)

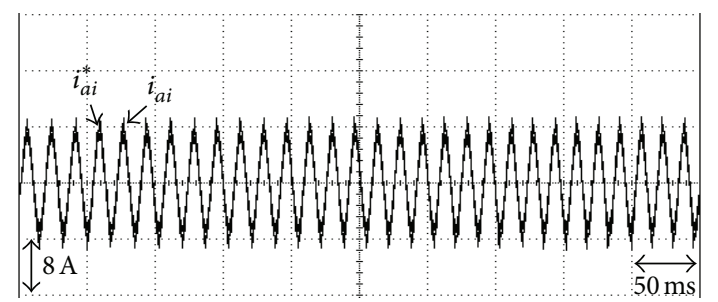

(d)

FIgURE 13: Experimental results of PMSM direct-driven PMSG system using the conventional NN controller for the $\Delta$ connection three-phase loads of $18 \Omega$ with $\omega_{r}=209.3 \mathrm{rad} / \mathrm{s}\left(n_{r}=2000 \mathrm{rpm}\right)$ at case 3: (a) rotor speed $\omega_{r}\left(n_{r}\right)$; (b) adjusting response of step desired magnitude $V_{d}^{*}$ of the DC bus voltage and actual measured magnitude $V_{d}$ of the DC bus voltage in output end of the rectifier; (c) adjusting response of step desired root-mean-square magnitude $V_{\mathrm{rms}}^{*}$ of the $\mathrm{AC} 60 \mathrm{~Hz}$ line voltage and actual measured root-meansquare magnitude $V_{\mathrm{rms}}$ of the AC $60 \mathrm{~Hz}$ line voltage in output end of the inverter $V_{\text {rms }}$; (d) tracking response of the desired phase current $i_{a i}^{*}$ and actual measured phase current $i_{a i}$ in phase $a i$ of the inverter.

modified Elman NN controllers were proposed to adjust the DC bus voltage of the rectifier and the $\mathrm{AC} 60 \mathrm{~Hz}$ line voltage of the inverter. In addition, the control performance of the proposed modified Elman NN controlled PMSG system direct-driven by PMSM is robust with regard to two operating conditions of the PMSG. Because of the weak robustness of the linear controller for the PI controlled PMSG system direct-driven by PMSM, dull DC bus voltage and AC $60 \mathrm{~Hz}$ line voltage adjusting responses are obviously obtained from 


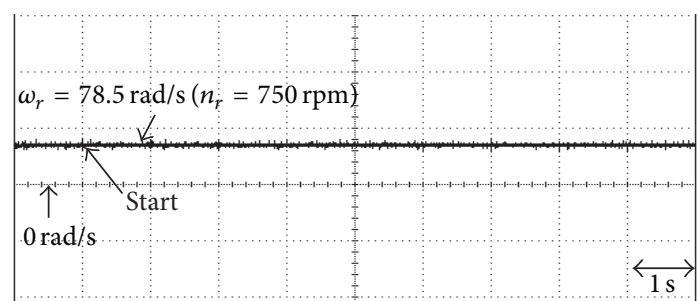

(a)

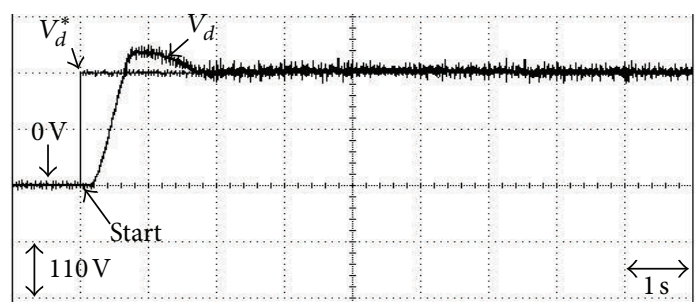

(b)

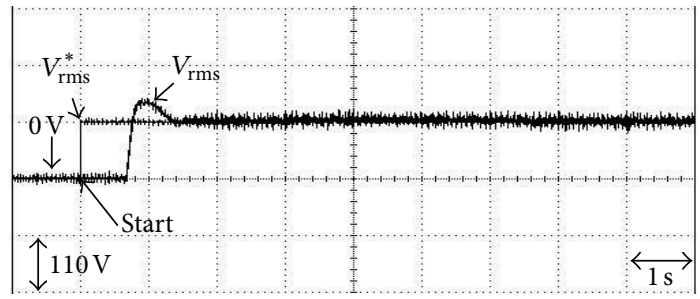

(c)

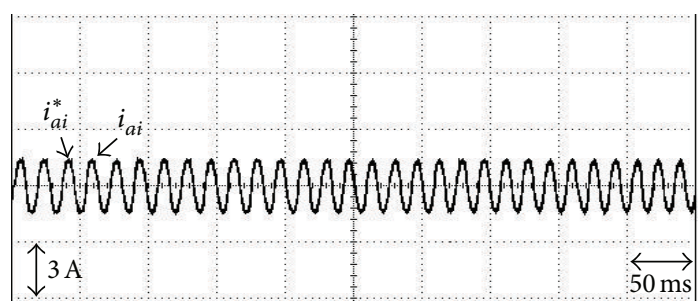

(d)

FIGURE 14: Experimental results of PMSM direct-driven PMSG system using the modified Elman NN controller for the $\Delta$ connection three-phase loads of $100 \Omega$ with $\omega_{r}=78.5 \mathrm{rad} / \mathrm{s}\left(n_{r}=750 \mathrm{rpm}\right)$ at case 1: (a) rotor speed $\omega_{r}\left(n_{r}\right)$; (b) adjusting response of step desired magnitude $V_{d}^{*}$ of the DC bus voltage and actual measured magnitude $V_{d}$ of the DC bus voltage in output end of the rectifier; (c) adjusting response of step desired root-mean-square magnitude $V_{\mathrm{rms}}^{*}$ of the AC $60 \mathrm{~Hz}$ line voltage and actual measured root-meansquare magnitude $V_{\text {rms }}$ of the AC $60 \mathrm{~Hz}$ line voltage in output end of the inverter $V_{\mathrm{rms}}$; (d) tracking response of the desired phase current $i_{a i}^{*}$ and actual measured phase current $i_{a i}$ in phase $a i$ of the inverter.

the experimental results. The important contribution of this study is successful application of the two sets of the same recurrent wavelet NN controllers on the PM synchronous motor direct-driven PM synchronous generator system to adjust the DC bus voltage of the rectifier and the AC $60 \mathrm{~Hz}$ line voltage of the inverter with robust control performance. Finally, control performance of the proposed modified Elman $\mathrm{NN}$ controller shown in experimental results is superior to the PI controller and the conventional NN controller for the

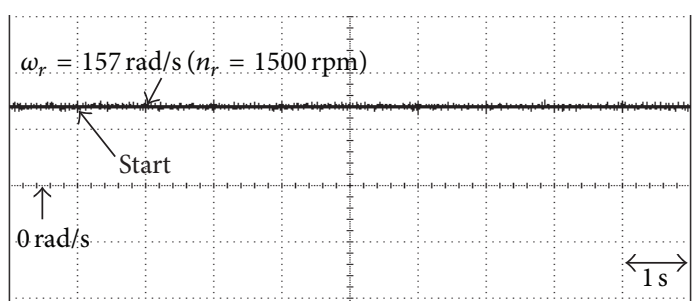

(a)

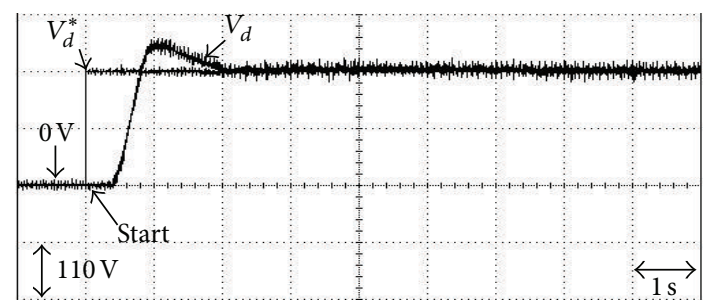

(b)

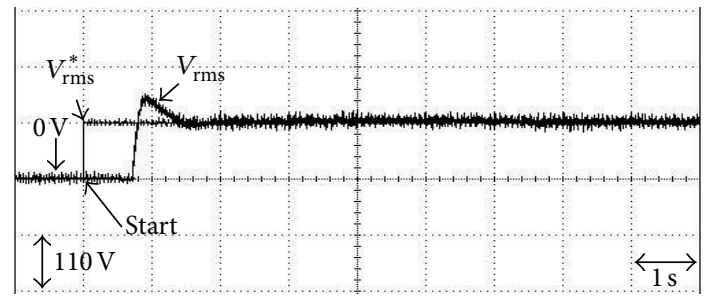

(c)

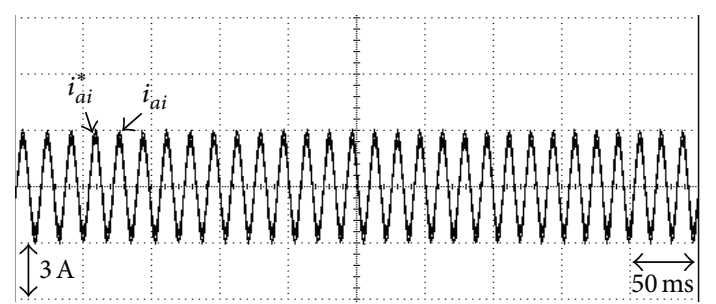

(d)

FIGURE 15: Experimental results of PMSM direct-driven PMSG system using the modified Elman NN controller for the $\Delta$ connection three-phase loads of $50 \Omega$ with $\omega_{r}=150 \mathrm{rad} / \mathrm{s}\left(n_{r}=1500 \mathrm{rpm}\right)$ at case 2: (a) rotor speed $\omega_{r}\left(n_{r}\right)$; (b) adjusting response of step desired magnitude $V_{d}^{*}$ of the DC bus voltage and actual measured magnitude $V_{d}$ of the DC bus voltage in output end of the rectifier; (c) adjusting response of step desired root-mean-square magnitude $V_{\mathrm{rms}}^{*}$ of the AC $60 \mathrm{~Hz}$ line voltage and actual measured root-meansquare magnitude $V_{\mathrm{rms}}$ of the AC $60 \mathrm{~Hz}$ line voltage in output end of the inverter $V_{\text {rms }}$; (d) tracking response of the desired phase current $i_{a i}^{*}$ and actual measured phase current $i_{a i}$ in phase $a i$ of the inverter.

PMSG system direct-driven by PMSM based on wind turbine emulator with a rectifier and an inverter for stand alone power applications.

\section{Acknowledgment}

The author would like to acknowledge the financial support of the National Science Council in Taiwan, through its Grant NSC 99-2221-E-239-040-MY3. 


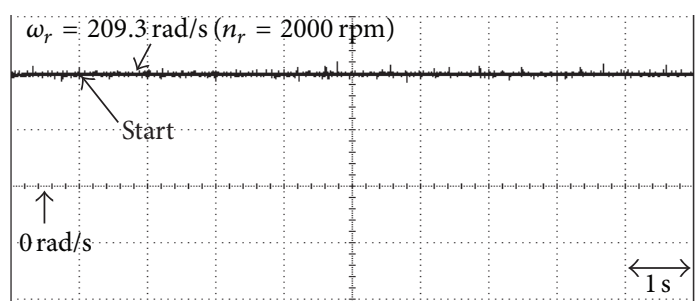

(a)

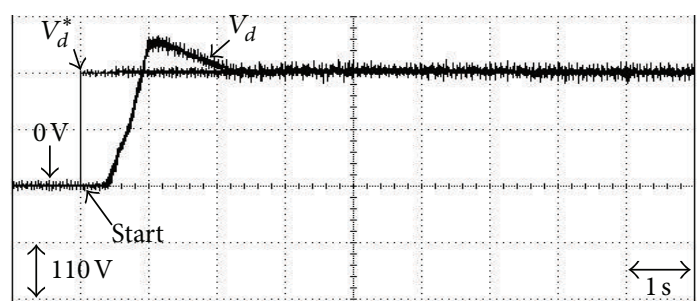

(b)

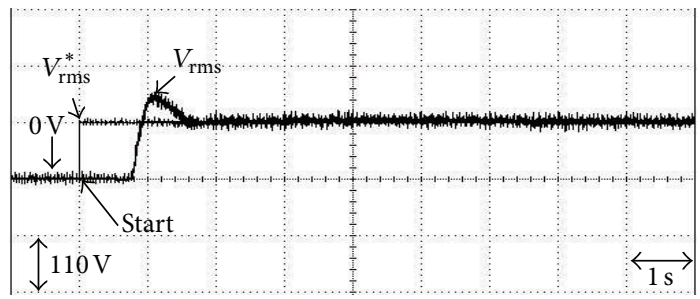

(c)

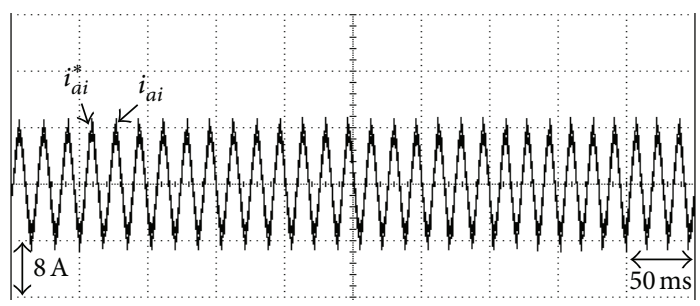

(d)

FIGURE 16: Experimental results of PMSM direct-driven PMSG system using the modified Elman NN controller for the $\Delta$ connection three-phase loads of $18 \Omega$ with $\omega_{r}=209.3 \mathrm{rad} / \mathrm{s}\left(n_{r}=2000 \mathrm{rpm}\right)$ at case 3: (a) rotor speed $\omega_{r}\left(n_{r}\right)$; (b) adjusting response of step desired magnitude $V_{d}^{*}$ of the DC bus voltage and actual measured magnitude $V_{d}$ of the DC bus voltage in output end of the rectifier; (c) adjusting response of step desired root-mean-square magnitude $V_{\mathrm{rms}}^{*}$ of the AC $60 \mathrm{~Hz}$ line voltage and actual measured root-meansquare magnitude $V_{\text {rms }}$ of the AC $60 \mathrm{~Hz}$ line voltage in output end of the inverter $V_{\text {rms }}$; (d) tracking response of the desired phase current $i_{a i}^{*}$ and actual measured phase current $i_{a i}$ in phase $a i$ of the inverter.

\section{References}

[1] K. Tan and S. Islam, "Optimum control strategies in energy conversion of PMSG wind turbine system without mechanical sensors," IEEE Transactions on Energy Conversion, vol. 19, no. 2, pp. 392-399, 2004.

[2] M. Kolhe, J. C. Joshi, and D. P. Kothari, "Performance analysis of a directly coupled photovoltaic water-pumping system," IEEE Transactions on Energy Conversion, vol. 19, no. 3, pp. 613-618, 2004.
[3] G. K. Andersen, C. Klumpner, S. B. Kjaer, and F. Blaabjerg, "A new green power inverter for fuel cells," in Proceedings of the IEEE 33rd Annual Power Electronics Specialists Conference (PESC '02), pp. 727-733, June 2002.

[4] Z. Lubosny, Wind Turbine Operation in Electric Power Systems, Springer, Berlin, Germany, 2003.

[5] T. Ackermann, Wind Power in Power Systems, John Wiley \& Sons, New York, NY, USA, 2005.

[6] M. Karrari, W. Rosehart, and O. P. Malik, "Comprehensive control strategy for a variable speed cage machine wind generation unit," IEEE Transactions on Energy Conversion, vol. 20, no. 2, pp. 415-423, 2005.

[7] I. Boldea, Synchronous Generators, Taylor \& Francis, Boca Raton, Fla, USA, 2006.

[8] M. Chinchilla, S. Arnaltes, and J. C. Burgos, "Control of permanent-magnet generators applied to variable-speed windenergy systems connected to the grid," IEEE Transactions on Energy Conversion, vol. 21, no. 1, pp. 130-135, 2006.

[9] S. Sajedi, F. Kahlifeh, T. Karimi, and Z. Khalifeh, "Maximum power point tracking of variable speed wind energy conversion system," International Journal of Physical Sciences, vol. 6, no. 30, pp. 6843-6851, 2011.

[10] F. Gharedaghi, H. Jamali, M. Deysi, and A. Khalili, "Maximum power point tracking of variable speed wind generation system connected to permanent magnet synchronous generator," International Review of Electrical Engineering, vol. 4, no. 4, pp. 10441049, 2011

[11] F. J. Lin, L. T. Teng, P. H. Shieh, and Y. F. Li, "Intelligent controlled-wind-turbine emulator and induction-generator system using RBFN," IEE Proceedings Electric Power Applications, vol. 153, no. 4, pp. 608-618, 2006.

[12] F. J. Lin, L. T. Teng, and M. H. Yu, "Radial basis function network control with improved particle swarm optimization for induction generator system," IEEE Transactions on Power Electronics, vol. 23, no. 4, pp. 2157-2169, 2008.

[13] J. L. Elman, "Finding structure in time," Cognitive Science, vol. 2, no. 14, pp. 179-211, 1990.

[14] M. N. Eskander, "Minimization of losses in permanent magnet synchronous motors using neural network," Journal of Power Electron, vol. 2, no. 3, pp. 220-229, 2002.

[15] M. Ghariani, I. B. Salah, M. Ayadi, and R. Neji, "Neural induction machine observer for electric vehicle applications," International Review on Modelling and Simulations, vol. 3, no. 3, pp. 314-324, 2010.

[16] P. Brandstetter, M. Kuchar, and I. Neborak, "Selected applications of artificial neural networks in the control of AC induction motor drives," International Review of Electrical Engineering, vol. 4, no. 3, pp. 1084-1093, 2011.

[17] R. Koker, "Design and performance of an intelligent predictive controller for a six-degree-of-freedom robot using the Elman network," Information Sciences, vol. 176, no. 12, pp. 1781-1799, 2006.

[18] A. Kalinli and S. Sagiroglu, "Elman network with embedded memory for system identification," Journal of Information Science and Engineering, vol. 22, no. 6, pp. 1555-1568, 2006.

[19] D. Samek, "Elman neural networks in model predictive control," in Proceedings of the 23rd European Conference on Modelling and Simulation, pp. 577-584, June 2009.

[20] F. J. Lin and Y. C. Hung, "FPGA-based elman neural network control system for linear ultrasonic motor," IEEE Transactions on Ultrasonics, Ferroelectrics, and Frequency Control, vol. 56, no. 1, pp. 101-113, 2009. 
[21] T. W. S. Chow and Y. Fang, "A recurrent neural-network-based real-time learning control strategy applying to nonlinear systems with unknown dynamics," IEEE Transactions on Industrial Electronics, vol. 45, no. 1, pp. 151-161, 1998.

[22] M. A. Brdys and G. J. Kulawski, "Dynamic neural controllers for induction motor," IEEE Transactions on Neural Networks, vol. 10, no. 2, pp. 340-355, 1999.

[23] X. D. Li, J. K. L. Ho, and T. W. S. Chow, "Approximation of dynamical time-variant systems by continuous-time recurrent neural networks," IEEE Transactions on Industrial Electronics, vol. 52, no. 10, pp. 656-660, 2005.

[24] C. H. Lu and C. C. Tsai, "Adaptive predictive control with recurrent neural network for industrial processes: an application to temperature control of a variable-frequency oil-cooling machine," IEEE Transactions on Industrial Electronics, vol. 53, no. 3, pp. 1366-1375, 2008.

[25] A. F. Payam, M. N. Hashemnia, and J. Faiz, "Robust DTC control of doubly-fed induction machines based on inputoutput feedback linearization using recurrent neural networks," Journal of Power Electron, vol. 11, no. 5, pp. 719-725, 2011.

[26] X. Z. Gao, X. M. Gao, and S. J. Ovaska, "Modified Elman neural network model with application to dynamical systems identification," in Proceedings of the IEEE International Conference on Systems, Man and Cybernetics, pp. 1376-1381, November 1996.

[27] X. Y. Yang, D. P. Xu, X. J. Han, and H. N. Zhou, "Predictive functional control with modified Elman neural network for reheated steam temperature," in Proceedings of the International Conference on Machine Learning and Cybernetics (ICMLC '05), pp. 4699-4703, June 2005.

[28] J. Li, H. Wu, and J. Pian, "The application of the equipment fault diagnosis based on modified Elman neural network," in Proceedings of the International Conference on Electronic and Mechanical Engineering and Information Technology, pp. 41354137, November 2011.

[29] M. Singh and S. Santoso, "Dynamic models for wind turbines and wind power plants," Subcontract Report NREL/SR-550052780, 2011.

[30] C. M. Lin, K. N. Hung, and C. F. Hsu, "Adaptive neuro-wavelet control for switching power supplies," IEEE Transactions on Industrial Electronics, vol. 54, no. 1, pp. 87-95, 2007. 


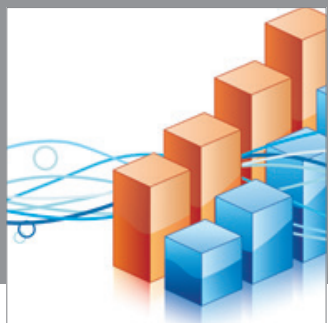

Advances in

Operations Research

mansans

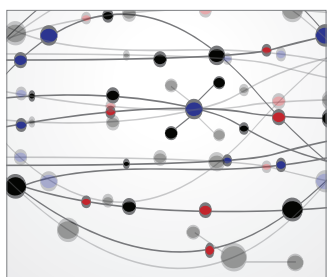

The Scientific World Journal
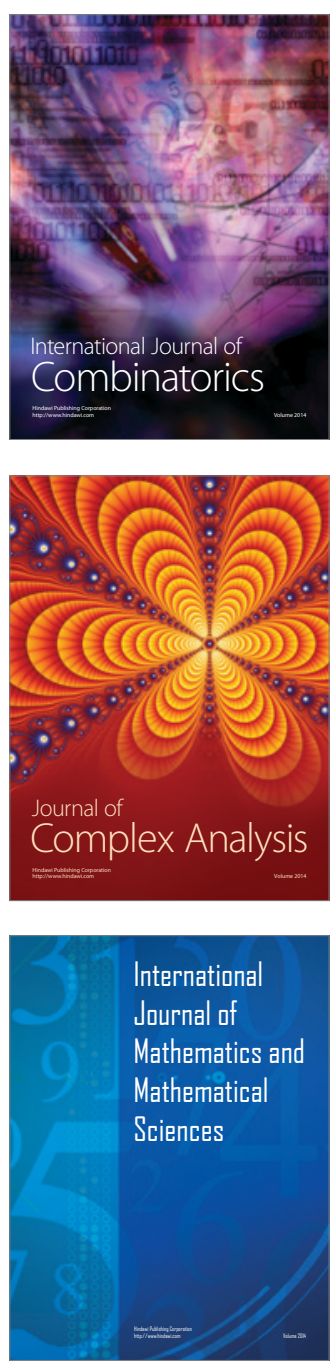
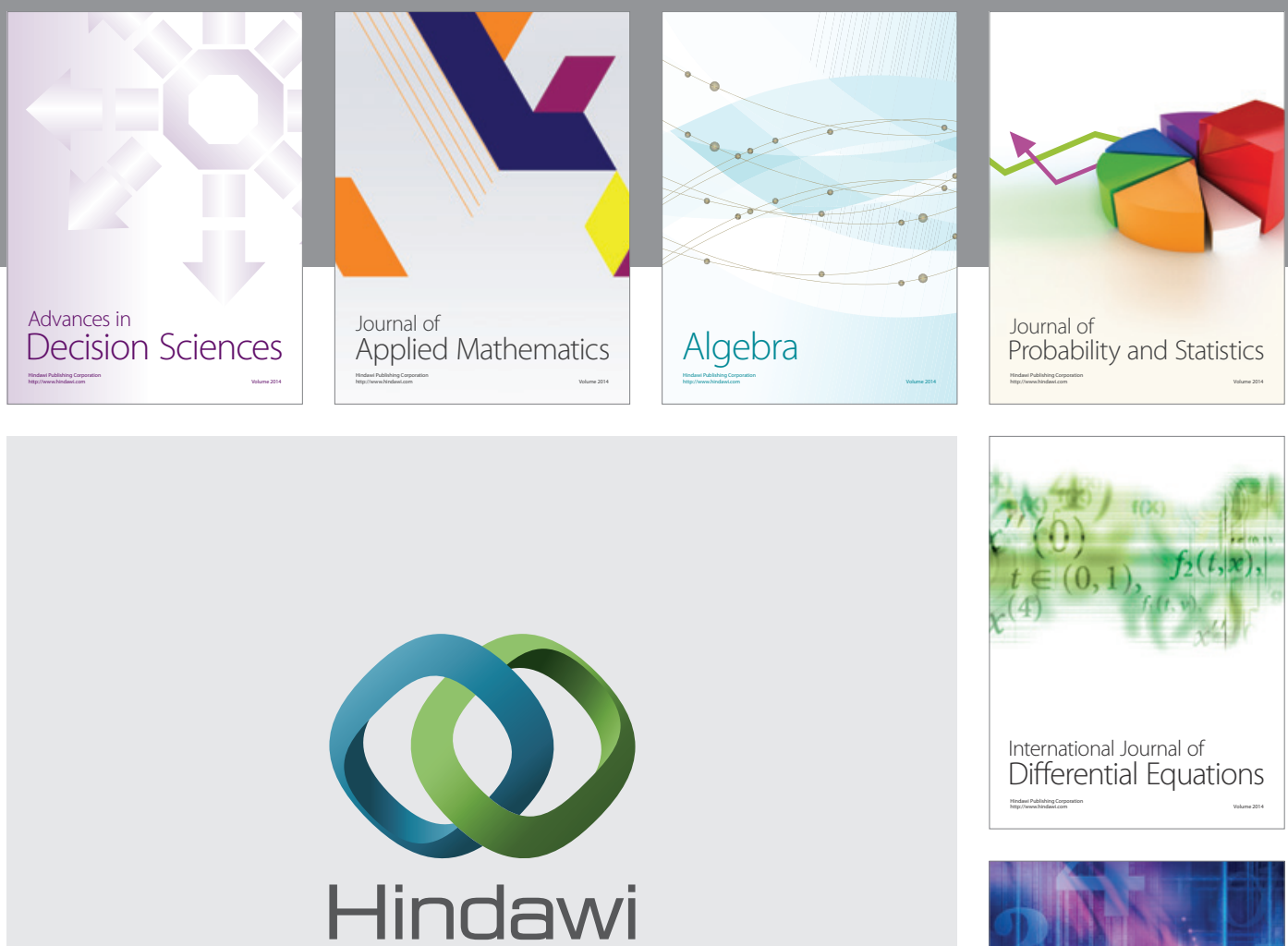

Submit your manuscripts at http://www.hindawi.com
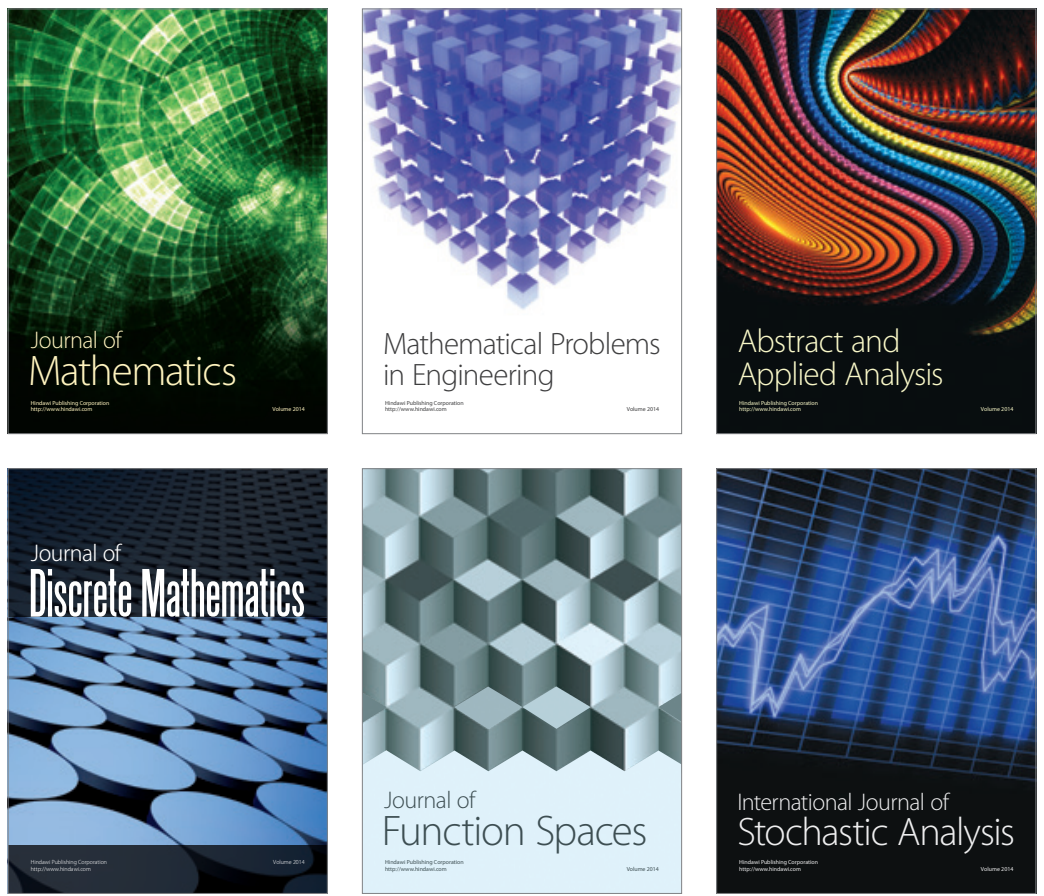

Journal of

Function Spaces

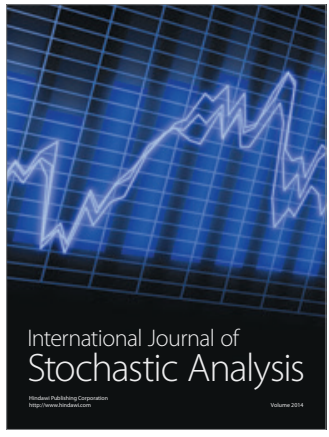

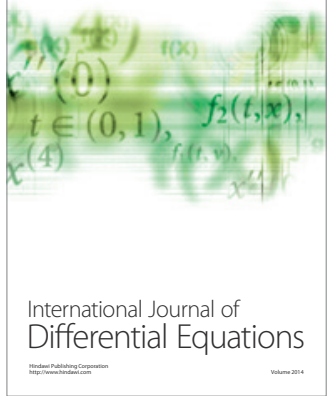
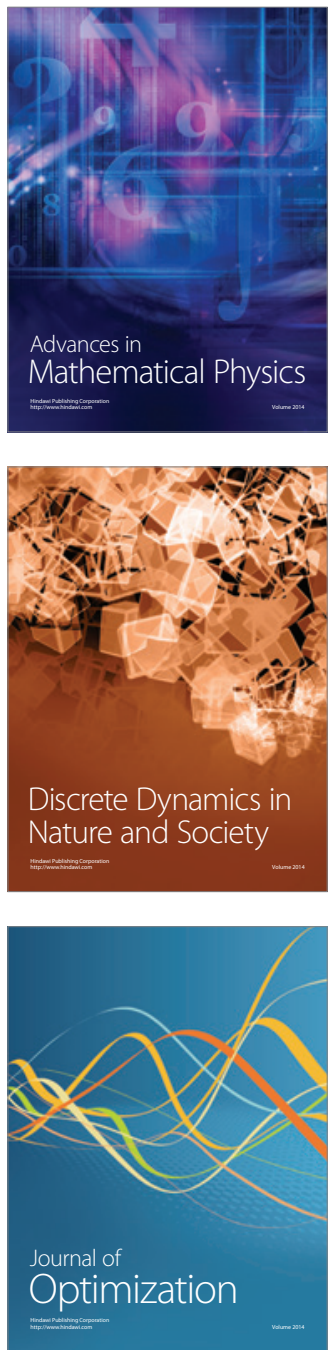